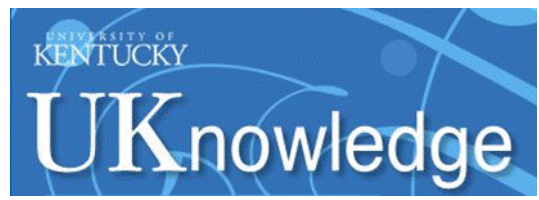

University of Kentucky

UKnowledge

Theses and Dissertations--Educational, School, and Counseling Psychology

Educational, School, and Counseling

Psychology

2013

\title{
Evaluating the Efficacy of Client Feedback in Group Psychotherapy
}

Norah C. Slone

norahslone@gmail.com

Right click to open a feedback form in a new tab to let us know how this document benefits you.

\section{Recommended Citation}

Slone, Norah C., "Evaluating the Efficacy of Client Feedback in Group Psychotherapy" (2013). Theses and Dissertations--Educational, School, and Counseling Psychology. 8.

https://uknowledge.uky.edu/edp_etds/8

This Doctoral Dissertation is brought to you for free and open access by the Educational, School, and Counseling Psychology at UKnowledge. It has been accepted for inclusion in Theses and Dissertations--Educational, School, and Counseling Psychology by an authorized administrator of UKnowledge. For more information, please contact UKnowledge@lsv.uky.edu. 


\section{STUDENT AGREEMENT:}

I represent that my thesis or dissertation and abstract are my original work. Proper attribution has been given to all outside sources. I understand that I am solely responsible for obtaining any needed copyright permissions. I have obtained and attached hereto needed written permission statements(s) from the owner(s) of each third-party copyrighted matter to be included in my work, allowing electronic distribution (if such use is not permitted by the fair use doctrine).

I hereby grant to The University of Kentucky and its agents the non-exclusive license to archive and make accessible my work in whole or in part in all forms of media, now or hereafter known. I agree that the document mentioned above may be made available immediately for worldwide access unless a preapproved embargo applies.

I retain all other ownership rights to the copyright of my work. I also retain the right to use in future works (such as articles or books) all or part of my work. I understand that I am free to register the copyright to my work.

\section{REVIEW, APPROVAL AND ACCEPTANCE}

The document mentioned above has been reviewed and accepted by the student's advisor, on behalf of the advisory committee, and by the Director of Graduate Studies (DGS), on behalf of the program; we verify that this is the final, approved version of the student's dissertation including all changes required by the advisory committee. The undersigned agree to abide by the statements above.

Norah C. Slone, Student

Dr. Robert J. Reese, Major Professor

Dr. Kenneth Tyler, PhD, Director of Graduate Studies 


\title{
EVALUATING THE EFFICACY OF CLIENT FEEDBACK IN GROUP PSYCHOTHERAPY
}

\section{DISSERTATION}

A dissertation submitted in partial fulfillment of the requirements for the degree of Doctor of Philosophy in the College of Education at the University of Kentucky

\author{
By \\ Norah Chapman Slone \\ Lexington, Kentucky \\ Director: Dr. Robert J. Reese, Ph.D., Associate Professor of Counseling Psychology \\ Lexington, Kentucky \\ 2013
}

Copyright $($ Norah Chapman Slone 2013 


\section{ABSTRACT OF DISSERTATION}

\section{EVALUATING THE EFFICACY OF CLIENT FEEDBACK IN GROUP PSYCHOTHERAPY}

A review of the adult psychotherapy outcome literature reveals decades of empirical studies that support the efficacy of group treatment for mental health concerns (Burlingame, Fuhriman, \& Mosier, 2003; Horne \& Rosenthal, 1997). Although research demonstrates positive results for the utilization of group therapy, between 33-50\% of clients drop out before treatment is completed (Baekeland \& Lundwall, 1975; Wierzbicki \& Pekarik, 1993). Methods for improving psychotherapy retention have been addressed through the application of a client feedback model (i.e., monitoring treatment outcome routinely). Although using client feedback has been found to be beneficial for improving retention and even enhancing client outcome in both individual (Lambert et al., 2001a; Lambert et al., 2002; Miller, Duncan, Brown, Sorrell, \& Chalk, 2006; Reese, Norsworthy, \& Rowlands, 2009; Whipple et al., 2003) and couples psychotherapy (Anker, Duncan, \& Sparks, 2009; Reese, Toland, Slone, \& Norsworthy, 2010), evidence of client feedback efficacy in a group format is limited but worthy of investigation given the positive individual and couples therapy findings. In the current study, I evaluated the effects of client feedback in group psychotherapy using a randomized clinical trial design. Participants $(N=85)$ attended psychotherapy groups that were randomly assigned to a client feedback (Feedback) or treatment as usual (TAU) condition. Clients in the Feedback condition were predicted to experience a statistically significantly higher prepost gain on the ORS compared to group clients in the TAU when controlling for their initial functioning. The effect size (ES) for Feedback between treatment conditions was found to be $d=0.35$ on the Outcome Rating Scale when initial treatment functioning was controlled, which is a medium ES (Cohen, 1988). Additionally, statistically significantly more clients in the Feedback condition experienced reliable and clinically significant change, attended statistically significantly more sessions, and dropped out at a lower rate than clients in the TAU condition. Results of this study were similar to those generated by other studies that evaluated the efficacy of client feedback with individuals (Reese et al., 2009) and couples (Anker et al., 2009; Reese et al., 2010). Study limitations and implications for future work are provided.

KEYWORDS: Group Psychotherapy, Client Feedback, Process Outcome, Continuous Assessment, Treatment Monitoring 
Norah C. Slone, M.S.

Student's Signature

June 25, 2013

Date 


\title{
EVALUATING THE EFFICACY OF CLIENT FEEDBACK IN GROUP PSYCHOTHERAPY
}

\author{
By
}

Norah Chapman Slone

Robert J. Reese, $\mathrm{PhD}$

Director of Dissertation

Kenneth Tyler, $\mathrm{PhD}$

Director of Graduate Studies

June 25, 2013

Date 


\section{Acknowledgments}

The following dissertation, while an individual work, benefitted greatly from the insights and direction of many people whom I have considered phenomenal mentors and supporters through this process. First, I would like to express deep gratitude for my Dissertation Chair, Jeff Reese, PhD. He has not only been an exceptional mentor in supporting me through this dissertation process but has contributed greatly to my personal and professional lives throughout both of my graduate programs. I am grateful for his endless patience towards my growth around research, particularly at every stage of this dissertation process. Next, I would like to thank my Dissertation committee and outside reader, Rory Remer, PhD., Sharon Rostosky, PhD., Brea Perry, PhD., and Michelle Martel, PhD. (outside reader). Your insights and support for my dissertation research, and me personally as a student, have provided a level of guidance that has substantially influenced my professional growth overall. I am also grateful to the participants in my study (who will remain anonymous for confidentiality purposes) for being willing to provide feedback and help us understand more about the efficacy of client feedback in group psychotherapy.

This project would not have been possible without the graciousness of the University of Kentucky Counseling Center. Many thanks to the Director, Mary Bolin, PhD., for being willing to support my project from an administrative perspective. I have the utmost gratitude for the Group Psychotherapy Coordinator, Susan Mathews-Duvall, $\mathrm{PhD}$ in this process, as her collaboration and advocacy for this study to the staff, trainees, and clients was invaluable. The unending support around coordination efforts and implementation was noteworthy, and I am grateful for the time and attention she provided in assisting with this project. 
I would also like to express my gratitude for Jonathan Kodet, a dear friend and colleague, for being willing to continue the weekly oversight of this project while I was on internship. His willingness to receive training on the research protocol as well as his reliable attention to the study throughout the Fall semester was remarkable.

In addition to the exemplary support of my mentors and research collaborators, I received equally exceptional support from my family and friends. I want to thank my entire family from the bottom of my heart for being supportive of me from the beginning of my graduate education, believing in me the whole way through, especially at times when I felt uncertain and waivered. Their support for me even when it took time away from them was something that I will never forget, as I know I would not be here without them. 
Acknowledgments ................................................................................................................................iii

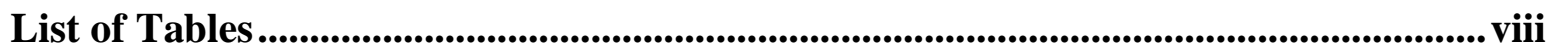

Chapter One: Introduction and Review of Selected Literature ...................................1

Efficacy of Group Therapy .....................................................................................1

Group Process and Outcome Research...........................................................................3

Therapeutic Alliance..........................................................................................

Therapist-client relationship.......................................................................5

Group alliance ...................................................................................................66

Negative Treatment Outcome .......................................................................................8

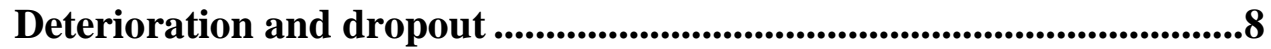

Clinical prediction...................................................................................10

Continuous Assessment ...............................................................................11

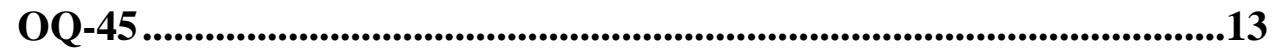

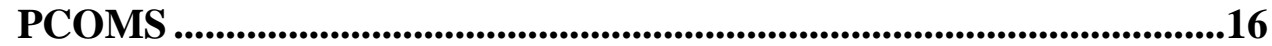

Individual therapy ................................................................19

Couples therapy........................................................................20

Strengths and limitations ...........................................................................21

Client feedback in group therapy .............................................................23

Purpose of This Study .....................................................................................................24

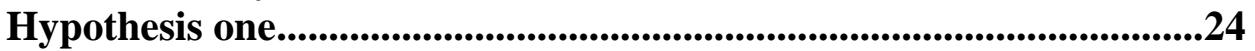

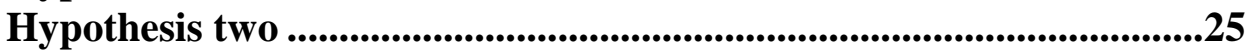

Hypothesis three......................................................................................25

Hypothesis four ............................................................................................25

Summary .............................................................................................................25

Chapter Two: Method ............................................................................................................27

Participants.......................................................................................................................227

Group Leaders...............................................................................................................227

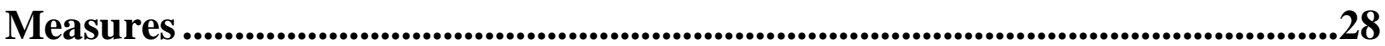

The Outcome Rating Scale (ORS; Miller \& Duncan, 2000) .................28

Center Assessment of Psychological Symptoms 34

(CCAPS-34; Locke et al., 2011) ..........................................................31

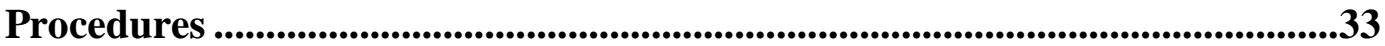

Informed consent …...................................................................................35

Group screening ............................................................................................35

Feedback intervention protocol procedures ..............................................36

Screening protocol .............................................................................36

Group session protocol...................................................................37

No change .......................................................................................38

Reliably deteriorated .....................................................38

Reliably changed ...................................................................39

Clinically significant change .............................................39 
TAU condition protocol procedures ..............................................................40

Screening protocol ...............................................................................40

Group session protocol ........................................................................40

CCAPS-34 ..................................................................................................40

Special considerations .........................................................................................41

Data Analyses .................................................................................................................41

Hypotheses one ..................................................................................................42

Hypothesis two ....................................................................................................44

Hypotheses three ......................................................................................................47

Hypotheses four .........................................................................................................48

Summary .............................................................................................................................49

Chapter Three: Results ............................................................................................50

Preliminary Analysis .................................................................................................54

Results of Hypotheses ..................................................................................................54

Hypothesis one .....................................................................................................54

Hypothesis two .................................................................................................58

Hypothesis three ......................................................................................................61

Hypothesis four .............................................................................................63

Summary ...........................................................................................................................66

Chapter Four: Discussion and Conclusions .....................................................................68

Efficacy of Client Feedback .........................................................................................68

Growth Rate ................................................................................................................70

Clinically Significant Change ......................................................................................70

Retention .........................................................................................................................71

Study Limitations ..............................................................................................................72

Naturalistic setting …………………..............................................................72

Intervention ......................................................................................................73

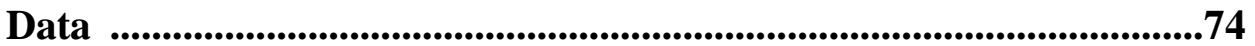

Implications and Future Recommendations ..............................................................76

Implications ...................................................................................................76

Future recommendations ..............................................................................77

Conclusions ......................................................................................................................8

Appendix A: Group Leader Demographic Form .................................................................81

Appendix B: Client Demographic Form ...............................................................................82

Appendix C: Computer Script ..............................................................................................84

Appendix D: Screening Script (Feedback condition) ..........................................................85

Appendix E: Screening Script (Control condition) ..............................................................87

Appendix F: Outcome Rating Scale ............................................................................88

Appendix G: Group Session Rating Scale ...........................................................................89

Appendix H: Graph for ORS and GSRS ............................................................................90

Appendix I: Counseling Center Assessment of Psychological Symptoms - 34 ........91

Appendix J: Signal Alarm Cards ............................................................................................93 


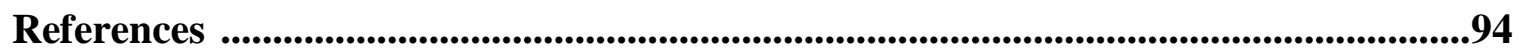

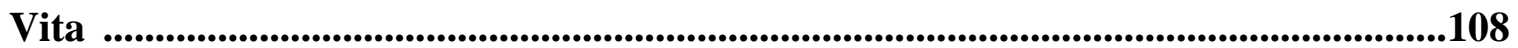




\section{List of Tables}

Table 3.1: Demographic Data for Client Participants $(N=85)$.........................................52

Table 3.2: Means, SD, and ES's for ORS and CCAPS-34 ..................................................55

Table 3.3: Results of Generalized Linear Model Predicting Post Group Treatment ORS Scores $(N=85)$

Table 3.4: Results of Generalized Linear Model Predicting Post Group Treatment CCAPS-34 Distress Index Scores $(N=76)$

Table 3.5: Fixed and Random Effect Estimates for Two-level Multilevel Growth Models for ORS $(N=85)$.

Table 3.6: Percentage of Clients who Achieved Clinically Significant Change in Feedback and TAU Conditions on the ORS $(N=85)$....

Table 3.7: Percentage of Clients who Achieved Clinically Significant Change in Feedback and TAU conditions on the CCAPS-34 Distress Index $(N=76)$ 


\section{Chapter One: Introduction and}

\section{Review of Selected Literature}

A review of the adult psychotherapy outcome literature reveals decades of empirical studies that support the efficacy of group treatment for mental health concerns (Burlingame, Fuhriman, \& Mosier, 2003; Horne \& Rosenthal, 1997). Although research demonstrates positive results for the utilization of group therapy, between $33-50 \%$ of clients drop out before treatment is completed (Baekeland \& Lundwall, 1975; Wierzbicki \& Pekarik, 1993). Moreover, Lambert and Ogles (2004) estimate that approximately 5$10 \%$ of clients deteriorate or worsen in psychotherapy. Methods for improving drop out and deterioration have been addressed through the application of a continuous assessment, or client feedback (i.e., monitoring treatment outcome routinely) approach. Using client feedback was developed to reduce the prevalence of premature termination (i.e., dropping out of therapy before "recovery" is achieved on an outcome measure) in psychotherapy (Lambert et al., 2001a; Lambert et al., 2002), but it has also been found to improve treatment outcome in both individual (Lambert et al., 2001a; Lambert et al., 2002; Miller, Duncan, Brown, Sorrell, \& Chalk, 2006; Reese, Norsworthy, \& Rowlands, 2009; Whipple et al., 2003) and couples psychotherapy (Anker, Duncan, \& Sparks, 2009; Reese, Toland, Slone, \& Norsworthy, 2010). Evidence of client feedback efficacy in a group format is limited but worthy of investigation given the positive findings with individual and couple therapy formats.

\section{Efficacy of Group Therapy}

Group psychotherapy outcome studies have demonstrated findings supporting the efficacy of this treatment format (Burlingame et al., 2003). In addition to its established efficacy, group therapy has been shown to be a more cost effective format of treatment 
and managed care organizations are encouraging its use in outpatient settings (Taylor, Burlingame, Fuhriman, Johansen, \& Dahl, 2001). Several studies presented in this review have demonstrated that group treatment is beneficial; however, fewer studies than in the individual psychotherapy literature utilize a randomized clinical trial (RCT) design and large sample size that also include process, client, or therapist variables known to predict client outcome (Antonuccio, Davis, Lewinsohn, \& Breckenridge, 1987; Chambless, Tran, \& Glass, 1997; Horvath \& Symonds, 1991; Lambert, 1992; Martin, Garske, \& Davis, 2000; Wampold, 2001).

Outcome studies have provided evidence of group psychotherapy efficacy for the treatment of various psychological concerns (Burlingame et al., 2003; Horne \& Rosenthal, 1997). Burlingame and colleagues (2003) conducted the only meta-analysis in the literature to date on the overall efficacy of group psychotherapy in a review of 111 published experimental and quasi-experimental studies with adults receiving treatment in outpatient settings (dissertations and studies with samples of couples, families, adolescents, and children were excluded). Results of this meta-analysis revealed an effect size (ES) of $d=0.58$ when group treatments were compared with waitlist controls. These findings indicated that group therapy clients were better off than $72 \%$ of untreated controls.

Group treatment has consistently been found to have comparable results to individual psychotherapy (Burlingame et al., 2003; McRoberts, Burlingame, \& Hoag, 1998). McRoberts and colleagues (1998) conducted a meta-analysis ( $N=23$ studies) on the available empirical research that compared both group and individual therapy for adults within the same study. The mean ES of 23 studies was $d=0.01$, which indicated 
that no treatment outcome differences were found for group versus individual therapy formats.

Although the efficacy of individual and group treatment formats is approximately equal, the methodology of the group psychotherapy outcome research has lagged behind the individual psychotherapy literature in three significant ways. First, group psychotherapy outcome studies are fewer in number. A reflection of this fact can be found by observing that many meta-analyses have been conducted on individual psychotherapy (e.g., Luborsky, Singer, \& Luborsky, 1975; Wampold et al., 1997); however, only one meta-analysis to date has been conducted for overall group therapy outcome studies that is not focused on a particular concern or diagnosis (Burlingame et al., 2003). A smaller sample of studies makes using meta-analytic methods to draw inferences from group research difficult for researchers. Second, much of the group therapy literature compares intervention groups to waitlist controls or placebo conditions. This type of methodology is no longer the current standard by which interventions are compared in the psychotherapy outcome literature (Lambert \& Ogles, 2004). Wellknown is the fact that psychotherapy compared to no treatment is effective (Burlingame et al., 2003; Smith, Glass, \& Miller, 1980). Instead, comparing intervention conditions to active treatments or Treatment as Usual (TAU) conditions is recommended (Lambert \& Ogles, 2004).

\section{Group Process and Outcome Research}

A third problem, and related to the limited number of group psychotherapy studies, is that less is known about the complex group psychotherapy processes that influence outcome in comparison to individual psychotherapy. The research on group process variables suggests that several therapeutic factors centered on the relationships in 
groups (i.e., alliance, cohesion, group climate) are significant predictors of outcome (Budman, Soldz, Demby, \& Feldstein, 1989; Gillaspy, Wright, Stokes, \& Adinoff, 2002; Johnson, Burlingame, Olsen, Davies \& Gleave, 2005; Joyce, Piper, \& Ogrodniczuk, 2007; Lindgren, Barber, \& Sandahl, 2008; Ogrodniczuk \& Piper, 2003; Ryum, Hagen, Nordahl, Vogel, \& Stiles, 2009). In fact, a recent publication from the Task Force on Evidenced-Based Therapy Relationships concluded that relationships in psychotherapy significantly benefit client outcome beyond the type of treatment delivered (Norcross \& Wampold, 2011). Task Force recommendations stated that practitioners should focus their efforts on cultivating strong therapeutic relationships and routinely monitor these relationships to improve outcomes and modify service delivery to avoid negative treatment outcome. One construct that describes relationships in both the individual and group therapy literature with strong empirical support is the therapeutic alliance.

\section{Therapeutic Alliance}

The therapeutic alliance is considered to be the most consistent predictor of client outcome next to clients' own strengths and resources they bring to therapy (Duncan, Miller, Wampold, \& Hubble, 2010; Horvath \& Symonds, 1991; Lambert, 1992; Lambert \& Ogles, 2004; Martin et al., 2000; Wampold, 2001). The therapeutic alliance has been found to predict as much as $30 \%$ of treatment outcome variance in the individual psychotherapy outcome literature (Duncan et al., 2010; Lambert \& Barley, 2001; Wampold, 2001). The effect of the alliance in individual psychotherapy outcome ranges in $\mathrm{ES}$ from $d=0.22$ to $d=0.26$ (Horvath \& Symonds, 1991; Martin et al., 2000; Wampold, 2001). Although the therapeutic alliance is a significant and consistent predictor of client success in individual psychotherapy, it is viewed as a more complex construct in group psychotherapy. 
Although several conceptualizations of the therapeutic alliance are found in the literature (see Greenson, 1965; Horvath \& Luborsky, 1993; Luborsky, 1976 for a comprehensive review), the most prevalent theory found in the group literature is that of Bordin (1979). He conceptualized the working alliance as an integrated relationship between therapists and clients developed in spite of the clients' pain and suffering (Bordin, 1979; Horvath \& Greenberg, 1986). Bordin purported that a strong relational bond and the agreement on therapeutic goals and tasks are pivotal to a positive working alliance.

Using Bordin's (1979) theory of the alliance has not only informed the conceptualization of the therapeutic alliance in groups, but also informed the subscales of a common working alliance measure found in the group therapy literature entitled the Working Alliance Inventory (WAI; Horvath \& Greenberg, 1986). The WAI is comprised of three subscales (i.e., Bond, Tasks, and Goals, also known as the tripartite model). Evidence from the literature suggests these elements of the working alliance are significant predictors of client treatment outcomes in group therapy across the types of relationships found within the group context, including both the therapist-client relationship (Abouguendia, Joyce, Piper, \& Ogrodniczuk, 2004; Piper, Ogrodniczuk, Lamarche, Hillscher, \& Joyce, 2005) and individual member's relationship to the groupas-a-whole, or group alliance (Gillaspy et al., 2002; Lindgren et al., 2008).

Therapist-client relationship. Findings for the therapist-client relationship in group psychotherapy are similar to those reported in the individual psychotherapy outcome literature (Horvath \& Symonds, 1991; Martin et al., 2000; Piper et al., 2005) given the definition and measurement of the relationship is similar across treatment formats. Research on the group therapist-client relationship has demonstrated that the 
client-rated alliance is a significant predictor of client change (Abouguendia et al., 2004; Budman et al., 1989; Joyce et al., 2007; Piper et al., 2005). For example, Piper and colleagues (2005) conducted a study with 107 treatment completers evaluating how the therapeutic alliance related to outcome in group therapy for complicated grief. The therapeutic alliance was assessed after every session by both the therapist and the client. Outcome variables included measures of grief symptoms, general symptoms, and severity of disturbance on target objectives of therapy and life satisfaction. They found the clientrated alliance to be a better predictor of outcome when compared to the therapist-rated alliance as well as statistically significant correlations with changes in general $(r=.24, p$ $<0.05)$ and grief symptom $(r=.23, p<0.05)$. Abouguendia and colleagues (2004) found that the client-rated alliance accounted for approximately $12 \%$ of the variation in outcome at the individual level and between $18-30 \%$ at the group level as a mediator of treatment expectancy and outcome. Last, Joyce and colleagues (2007) found that the client-rated alliance significantly predicted client scores on all five outcome measures in their study.

Group alliance. Group alliance (defined as an individual client's relationship to the group-as-a-whole) outcome studies are fewer in number (Lindgren et al., 2008); however, the group alliance is considered a consistently modest predictor of client outcome in groups (Budman et al., 1989; Gillaspy et al., 2002; Lindgren et al., 2008; Marziali, Munroe-Blum, \& McCleary, 1997). For example, Lindgren and colleagues (2008) investigated whether the group alliance predicted outcome with 29 women and 11 men randomized to a short-term group psychodynamic, group cognitive treatment, or TAU for burnout-related depression. The group alliance was measured using the California Psychotherapy Alliance Scales (CALPAS-G; Gaston \& Marmar, 1994), a 
measure of the therapeutic alliance adapted for group use. Results from a multivariate analysis of variance indicated that mean alliance scores significantly predicted reductions in anxiety and global symptoms, but not depression. Group alliance explained $50-55 \%$ of the variance in change scores on measures of global symptoms and anxiety and $22 \%$ of the change on depression outcome scores. The amount of variance in outcome explained by the group alliance in this study is similar to variance estimates found for the alliance in the individual therapy literature (36-57\%; Gaston, Marmar, Gallagher, \& Thompson, 1991).

Although the alliance is known as an important contributor to client change in group therapy, alliance-outcome research studies found in group psychotherapy have four primary limitations. First, the measurement of the group alliance is problematic due to the lack of a clear operationalization of the construct. This problem is observed with the use of non-uniform inclusion of who and what constitutes the group alliance. Group alliance studies have varied in whether the alliance is comprised of the therapist-client relationship, group member relationships, or a combination of both relationships. Moreover, a distinction between group alliance and other group processes is lacking. For example, the CALPAS-G has been used to measure both group alliance and cohesion in the literature, which threatens external validity and creates confusion when generalizing findings (Crowe \& Grenyer, 2008; Lindgren et al., 2008). The lack of uniform definition of the group alliance makes adequately evaluating and contrasting this construct with other process variables difficult. A second weakness is the samples in many of the alliance and outcome studies are small, with few studies exceeding a sample size of 100 , which may be problematic with regards to whether adequate power has been achieved to reject the null hypothesis. Third, statistical analyses used in some studies do not account 
for the interdependent nature of group-level data (Budman et al., 1989; Piper et al., 2005). Failure to account for interdependent data, or individual data observations within clusters that may reflect mutual dependence on one another, increases the likelihood of Type I error (Raudenbush \& Bryk, 2002). Last, group alliance-outcome studies fail to account for therapist effects, as recommended in the general psychotherapy outcome literature (Lambert \& Ogles, 2004; Wampold \& Brown, 2005). Doing so helps determine whether variation in client outcome is attributable to differences among group leaders. Failure to do so may be overestimating the variance attributed to individual or group levels.

\section{Negative Treatment Outcome}

Although the efficacy of group psychotherapy has been well documented, discussed less in the group therapy outcome research is the risk that all clients do not progress as expected, remain in treatment through its intended duration, or develop positive relationships in the group (Baekeland \& Lundwall, 1975; Burlingame et al., 2003; Hoffmann, Gleave, Burlingame, \& Jackson, 2009; Wierzbicki \& Pekarik, 1993). In addition, the literature also reports an often overlooked problem that clinicians are poor judges of which clients are getting worse in treatment or at risk of dropping out (Chapman et al., 2012; Hannan et al., 2005).

Deterioration and dropout. Psychotherapy outcome research has shown that a significant number of clients do not make progress as expected in treatment and, as a result, are at risk for deterioration and drop out (Lambert \& Ogles, 2004). A recent metaanalysis suggested that clients in a Feedback condition deteriorate at a rate of approximately 4-5\% (Lambert et al., 2005), which is an improvement over deterioration rates in the overall psychotherapy outcome literature (5-10\%; Lambert \& Ogles, 2004). Moreover, approximately $47 \%$ of individual therapy clients drop out of therapy all 
together (Baekeland \& Lundwall, 1975; Wierzbicki \& Pekarik, 1993). These rates do not differ significantly between individual and group therapy formats (Baekeland \& Lundwall, 1975; Davis, Hooke, \& Page, 2006). Although the reality that not all clients improve is disconcerting, research provides some preliminary information about clients who are at risk for negative treatment outcome in group therapy.

Two recent studies provided data on the characteristics of clients who tend to drop out or deteriorate in group therapy (Davis et al., 2006; Hoffmann et al., 2009). In a qualitative study by Hoffmann and colleagues (2009), the authors utilized group members $(N=14)$ from a larger sample who showed either significant improvement or deterioration in group therapy. Client characteristics were analyzed using a Hermeneutic qualitative approach, which allows researchers to interpret meaning of parts within a larger context, such as a psychotherapy group. Results indicated that several characteristics could be noted for each of the deterioraters and improvers. First, deterioraters tended to self-disclose information early on in therapy and expected others to do the same. Results suggested that these same members tended to share less as the group progressed. Second, members who deteriorated tended to praise the progress of the group early in therapy more than others. Finally, members who deteriorated spent more time giving feedback to others rather than focusing on their own concerns that brought them to group. In addition, Davis and colleagues (2006) noted that clients who were younger, had low self-esteem, high neuroticism traits, affective disorders, and poorer relationships were more likely to drop out of group CBT treatment.

Conversely, Hoffmann and colleagues (2009) found that group members who improved tended to be more cautious about self-disclosures. Improvers also accepted positive feedback and time offered to discuss their concerns in the group. Characteristics 
of clients noted in these studies suggest that group leaders should monitor and intervene early with members who exhibit these characteristics to prevent treatment failure.

However, research studies (e.g., Chapman et al., 2012; Hannan et al., 2005) have shown this task may be difficult to complete accurately given that clinicians are generally poor judges of which clients are more likely to drop out or deteriorate in individual and group treatment.

Clinical prediction. In a study conducted by Hannan and colleagues (2005), therapists were asked to identify clients in their caseloads who may be deteriorating. Therapists' predictions were compared with predictions made by a statistical algorithm associated with the Outcome Questionnaire-45 (OQ-45; Lambert et al., 1996) computer software program. Clients $(N=550)$ being treated at a university counseling center responded to the OQ-45 before every session to monitor outcome. Therapists in the study were not privy to their clients' scores during the time of the study. Out of a total of 944-recorded predictions, only three therapist-predictions were made that suggested clients in their caseloads were deteriorating at the end of a three-week period. When OQ45 outcome scores were analyzed, 40 clients actually deteriorated. Therapists only successfully predicted 1 out of 40 cases, while the OQ-45 signal alarm system successfully predicted 36 out of 40 cases. This large discrepancy highlights the possibility that a formal method of monitoring client outcome may help to identify clients who are at-risk of dropping out of treatment. It provides the therapist an opportunity to change the course of treatment.

A recent study by Chapman and colleagues (2012) found that a similar difficulty occurs with clinical prediction in group therapy. A total of 14 group leaders providing services in 10 groups comprised of 64 clients recruited from a university counseling 
center $(n=33)$ and an inpatient psychiatric hospital $(n=31)$ participated in the study. Only 49 participants completed post-test data in this sample. Each group leader predicted the final outcome of their group members after the third group session by sharing their belief as to whether a member would be reliably improved, have no significant change, or be reliably worse according to clients' change from pre to post test on the OQ-45 (Lambert et al., 1996). Therapists predicted that 31 of 49 clients would demonstrate reliable improvement; however, only $13 \%$ (or 4 out of 31 clients) of their predictions were accurate. No therapist accurately predicted clients who were reliably worse in their groups $(n=10)$. Although the sample size for this study was small, the results further support the need for formalized methods of monitoring outcome in group therapy.

\section{Continuous Assessment}

The practice of continuously assessing client outcome across treatment, also known as client feedback, has been proffered as a way to more accurately identify clients who are at risk for negative treatment outcome. This concept emerged from two seminal research studies that evidenced a rationale for monitoring individual treatment outcome to improve recovery and retention in psychotherapy.

The first study established that clients who remain in therapy longer experience greater benefits in treatment. The seminal work of Howard, Kopta, Krause, and Orlinsky (1986) demonstrated that a dose (i.e., number of sessions) and effect (i.e., the unit normal deviation of the normal distribution related to a percentage of clients who have been judged to improve) relationship exists in psychotherapy, meaning that a higher percentage of clients who remained in therapy for more sessions were measurably improved. For example, approximately $50 \%$ of clients were stated to be measurably 
improved after eight sessions in a sample of $N=2,400$ clients. Approximately $75 \%$ clients were measurably improved by session 26 .

A second study further explored how client outcome was affected by individual differences and presenting concerns, particularly within a paradigm shift in the field that affected clients' accessibility to particular "doses" of psychotherapy (i.e., managed care, rising costs of psychotherapy). Howard, Moras, Brill, Martinovich, and Lutz (1996) observed client change across treatment and found that client's expected change in therapy may deviate from the average "effect" given their presenting characteristics and initial functioning. Hansen, Lambert, and Foreman (2002) further demonstrated that clients in naturalistic settings changed at variable rates and a significant portion (20\%) dropped out of therapy before achieving recovery. Therefore, Howard et al. (1996) called for a systematic change in the methodology of evaluating psychotherapy outcome research to monitor when clients are deviating from their expected course in treatment. They proposed that outcome studies shift to focus on individual client change in naturalistic settings (i.e., effectiveness research) rather than on clinical efficacy (i.e., prepost research in highly controlled labs) to improve client outcome and generalizability of findings.

The concept of client feedback was developed from this paradigm of clientfocused research to monitor clients' observed change in psychotherapy (Howard et al., 1996). Seminal work by Lambert, Hansen, and Finch (2001b) demonstrated how this concept of monitoring client feedback can facilitate clinical work through the use of a brief measure of client global psychological functioning used each session to identify clients who are not making progress in therapy as expected. Using client feedback in clinical practice was hoped to identify such clients more quickly to improve retention as 
well as to assist clinicians in making treatment decisions that benefit their clients' outcomes, given their presenting concerns and individual differences. Effectiveness of such formalized systems for monitoring client outcome has been evaluated in the literature.

Client feedback has been found to improve retention in psychotherapy and enhance client treatment outcome (Lambert et al., 2001a; Miller, Duncan, Sorrell, \& Brown, 2004). A review of the research on formal feedback systems yielded empirical evidence for two primary systems used in the U.S.: The OQ-45 System (Lambert et al., 1996) and the Partners for Change Outcome Management System (PCOMS; Miller et al., 2004). Lambert and Shimokawa (2011) conducted a recent meta-analysis on the overall effectiveness of client feedback for each system. Findings on the effectiveness of PCOMS were based on three well-designed randomized clinical trial (RCT) studies that yielded an overall ES of $r=.23$ for clients in a Feedback condition versus TAU. Metaanalytic results of six RCT studies using the OQ-45 System yielded an overall ES of $r$ $=.25$ for clients in a Feedback condition not progressing as expected, or not on track (NOT). Findings from the empirical research evaluating each system will be provided to establish a greater understanding of how using client feedback has been evaluated in regards to treatment outcome in psychotherapy.

OQ-45. Lambert and colleagues (1996) developed a formal system of client feedback to feasibly monitor treatment outcome every session in hopes of enhancing outcome by preventing clients from deterioration and drop out. This system is comprised of a 45-item outcome measure of global psychological functioning across domains of Symptom Distress, Interpersonal Relationships, Social Roles, and Overall Sense of Wellbeing. The OQ-45 has an accompanying computerized signal alarm system that uses 
statistical algorithms to project client change trajectories. The algorithms provide an automated signal to therapists alerting them to their clients' progress so they may intervene early with clients who are NOT.

The efficacy of the OQ-45 System has been evaluated in six large RCTs. Lambert and colleagues (2001) first demonstrated using the OQ-45 as a method for obtaining client feedback in therapy improves client outcome, specifically with clients who are NOT. Clients $(N=609)$ in therapy at a university counseling center were randomized to four groups: NOT in a Feedback condition or a TAU condition or on track (OT; making progress as expected) in a Feedback or TAU condition. Lambert and colleagues found that all clients in a Feedback condition improved on average of 4.99 OQ-45 points, but clients who began treatment in the dysfunctional range (an OQ-45 score of 64 or above) achieved even greater gains with an average of 7.99 OQ-45 point improvement over the course of therapy. Of clients who began treatment in the dysfunctional range, $11.2 \%$ achieved clinically significant change (reliable change that includes moving from the clinical to nonclinical range) after 3.3 sessions. In addition, an ES of $d=0.44$ for feedback was only statistically significant for NOT clients, which is a medium ES according to Lipsey's (1990) standards. No statistically significant differences were noted for OT clients in the Feedback condition compared to TAU. Clients who were NOT in the Feedback condition also attended significantly more sessions (9.68 versus 5.03) and had a statistically significantly lower deterioration rate (e.g., 14 OQ-45 point reduction in scores from pre to post; $6 \%$ versus $23 \%$ ) than NOT clients in a TAU condition.

Findings related to client outcome were successfully replicated in another largescale study with an independent sample of $N=1,020$ clients from a university counseling 
center (Lambert et al., 2002). An ES similar to the original study was found $(d=0.40$ versus $d=0.44$ ) and feedback effects were only significantly different than TAU for NOT clients. Clients who were in a Feedback condition (both NOT and OT) were found to have attended statistically significantly more sessions in this study when compared to clients in a TAU condition (both NOT and OT). Although these results are impressive for treatment outcome, statistically significant differences between the Feedback and TAU condition were only found for NOT clients. Mixed results for session attendance were found between these studies, with greater attendance noted for either NOT clients in a Feedback condition or for all clients in a Feedback condition.

Incorporating clinical support tools (CST's; Slade et al., 2008; Whipple et al, 2003), in addition to client feedback, has also enhanced client outcomes and prevented deterioration and drop out. Whipple and colleagues (2003) defined CST's as empirically supported problem solving tools (i.e., assessments measuring motivation, therapeutic alliance, social support, and perfectionism) and resources (i.e., diagnostic clarification, medication referrals) provided to therapists as part of a decision tree that accompanies the signal alarm system described above to more fully address NOT clients' needs based on their OQ-45 responses. Clients $(N=981)$ from a university counseling center participated in 1 of 5 treatment conditions: NOT clients received either feedback, TAU, or feedback plus CST's and OT clients received feedback or TAU. Results of this study indicated that clients who were NOT and received feedback plus CST's had statistically significantly better outcome than those NOT clients who received feedback or TAU. An ES of $d=$ 0.70 was found for clients who were NOT and received feedback plus CST's. Clients who were NOT and provided CST's and feedback also attended significantly more sessions (12.02 versus 7.96$)$ and had a statistically significantly lower deterioration rate $(8.5 \%$ 
versus $19.1 \%$ ) compared to clients in a NOT TAU condition. Slade and colleagues (2008) found similar results in a replication study with a sample of university counseling center clients $(N=1,101)$; however, clients in a Feedback condition overall attended statistically significantly more (1.5 sessions more) sessions than those in a TAU condition. Again, mixed results were noted for session attendance, with greater attendance noted for either NOT clients in a CST and Feedback condition or for all clients in a Feedback condition.

Client feedback studies have also demonstrated that to whom feedback is directed could make a difference in treatment outcome. Two studies yielded mixed findings when client outcome was compared across conditions in which feedback via the OQ-45 was given to therapists only or to both therapists and clients (Harmon et al., 2007; Hawkins, Lambert, Vermeersch, Slade, \& Tuttle, 2004). Hawkins and colleagues (2004) utilized participants from a community sample who were randomized to one of three conditions: feedback to therapists only, feedback to both therapists and clients, or to TAU (archival data). Findings from this study indicated outcomes were significantly improved when both therapists and clients received feedback on their progress every session than when compared to therapist-only feedback. Conversely, Harmon and colleagues (2007) found that treatment outcomes in a sample of university students $(N=1,374)$ were not enhanced when clients also viewed the feedback. Results of Shimokawa, Lambert, \& Smart's (2010) mega analytic (i.e., approach for calculating pooled ES's with a large $N$ ) study suggested conclusively that providing feedback to both the therapist and the client does not add statistically significant benefit to providing feedback to the therapist only.

PCOMS. The Partners for Change Outcome Management System (PCOMS; Miller et al., 2004) is a formal client feedback system developed to monitor both treatment outcome and alliance each session to prevent deterioration and drop out, 
primarily for those who are at risk of premature termination from therapy. PCOMS was designed as a client feedback intervention that is feasible for practitioners and is comprised of two ultra brief measures of client outcome and the therapeutic alliance (for use in individual and couple therapy) or the group alliance (for use in group psychotherapy) that are administered every session (Miller \& Duncan, 2004). The Outcome Rating Scale (ORS; Miller \& Duncan, 2000) is a 4-item instrument that uses a visual analog scale to provide a measure of global psychological functioning and is administered before each session begins. The 4-items on the ORS are the subscales of the OQ-45 that measure global psychological functioning across Individual (i.e., personal well-being), Interpersonal (i.e., family, close friends), Social (i.e., work, school), and Overall (i.e., general sense of well-being) domains. The Session Rating Scale (SRS; Miller, Duncan, \& Johnson, 2000) is a 4-item instrument that also uses a visual analog scale to provide a measure of the therapeutic alliance and is administered at the end of every session. The SRS measures the Bond, degree of agreement on Goals and Topics, degree of agreement on Approach or Method, and an Overall rating of the alliance between the therapist and client. The first three items, (e.g., Bond, Goals and Topics, and Approach or Method) were conceptualized and operationally defined using Bordin’s (1979) theory of the therapeutic alliance. The final item, the Overall rating, was developed to measure the confident collaboration dimension of the alliance (Hatcher $\&$ Barends, 1996), or the client's overall rating of the session. The ORS and SRS can be administered, scored, and interpreted in approximately one minute each. Total scores are plotted on a graph to show client progress over the course of therapy (see Methods section for further administrative procedures). Therapists are able to monitor client progress on this graph during each session and discuss with clients what changes are 
being made that are either supporting their improvement or to determine what is needed from their perspective to help them feel improved in regards to their progress and/or the therapeutic alliance.

Similar to the OQ-45 (Lambert et al., 1996), PCOMS has an accompanying method for signaling therapists that their clients are "at risk (i.e., clients who have not made clinically significant progress $[\geq 5$ ORS points improvement from baseline, or session 1] on the ORS or have deteriorated in therapy [ $\geq 5$ ORS points worsened from baseline, or session 1] by session 3 or session 6)" of prematurely terminating (dropping out of therapy before reaching "recovery" [i.e., $\geq 5$ ORS points improved from baseline and across the clinical cutoff of 25 on the ORS; Miller \& Duncan, 2004]) from treatment. Miller and Duncan (2004) suggest that clinicians with clients who are noted to be at risk of premature termination incorporate client feedback received on these measures. One way to do this is by having a more overt discussion of the client's overall treatment progress to determine if modifications to the therapeutic approach and/or therapeutic alliance or adjunctive treatment in the form of psychiatric medication or other services are needed to help improve client outcome that session.

Given the rationale that similar concerns with deterioration, drop out, premature termination, and difficulties with clinical prediction occur across treatment formats, including group therapy, (Chapman et al., 2012; Hannan et al., 2005; Lambert \& Ogles, 2004), PCOMS measures have also been developed and recommended for group psychotherapy by utilizing both the ORS and a measure of the group alliance (member's relationship to the group-as-a-whole) called the Group Session Rating Scale (GSRS; Duncan \& Miller, 2007). Both measures are administered, scored, plotted on a graph, and interpreted in the same way as has been described above with the ORS and SRS. 
Although these measures have been developed and recommended for use in group therapy, no published research to date has been developed for implementing client feedback in a group format. However, the rationale for extending this intervention to a group format is warranted given that PCOMS' efficacy has been demonstrated through three well-designed RCT studies with both individual and couple psychotherapy.

Individual therapy. The effectiveness of PCOMS was first examined over a 2year period with a sample of $N=6,424$ clients who utilized telephone-based counseling as part of an employee assistance program (Miller et al., 2006). Evaluation of this system took place across four phases beginning with initial training on how to implement PCOMS for six months, followed by a baseline evaluation of TAU using the ORS and SRS for the next six months. The third phase included the provision of a signal warning system in which therapists were provided feedback before a final fourth phase of data collection was completed by evaluating feedback and outcome. Effectiveness of therapy improved from $d=0.39$ at baseline to $d=0.79$ at final evaluation. Results of this study demonstrated psychotherapy outcome improved significantly as a result of using PCOMS to systematically obtain client feedback with a medium to large ES, according to Cohen's (1988) standards. In addition, Miller and colleagues (2006) found clients whose therapeutic alliance was monitored across treatment demonstrated better outcomes, as evidenced by the fact that three times as many clients who responded to the SRS after the first session returned for a second session (19\% versus 6\%). Results of this initial study demonstrated that using PCOMS is feasible and effective for improving client outcome and retention.

Results of Miller and colleagues' (2006) study were successfully replicated in two independent RCT studies conducted by Reese and colleagues (2009). Two independent 
samples $(N=74$ in each study) from a university counseling center and a graduate training clinic that served the community were used to compare clients who were randomized to a PCOMS or TAU condition. Results of this study indicated that clients in the PCOMS conditions improved twice as much as clients in TAU conditions overall. More clients in the PCOMS conditions also achieved statistically significantly higher rates of reliable change (defined as 5 point improvement on the ORS; Study 1: $80 \%$ versus 54.2\%; Study 2: $66.67 \%$ versus $41.4 \%)$. Medium $(d=0.49)$ to large effect sizes $(d=0.54)$ were found in favor of using feedback with clients in psychotherapy. In addition, no statistically significant differences were noted in either the number of sessions attended or in deterioration rates across treatment conditions in either study. Reese and colleagues successfully replicated the results of Miller and colleague's study using the RCT methodology to demonstrate that PCOMS improves psychotherapy for all clients.

Couples therapy. Though using PCOMS with individual clients has yielded impressive results, similar results have been found with couples (Anker et al., 2009; Reese et al., 2010). Anker and colleagues (2009) investigated the effectiveness of client feedback using PCOMS with 205 heterosexual Euro-Scandinavian couples randomized to 10 therapists in either a feedback or TAU condition. Results of hierarchical linear modeling analyses showed couples in the PCOMS condition experienced significantly improved outcomes compared to couples in the TAU condition. A medium to large ES of $d=0.50$ and gains of approximately 8.58 points on the ORS at post-test were found for couples in the PCOMS condition compared to 3.64 points in the TAU condition. Moreover, a large percentage of couples in the PCOMS condition achieved clinically significant change $(40.8 \%)$ by the end of treatment compared with $10.8 \%$ of couples in 
the TAU condition. Significantly more couples in the PCOMS condition $(81.59 \%$ versus $65.75 \%$ ) remained together at 6-month follow up.

Findings of Anker and colleagues' (2009) research were replicated in a study conducted by Reese and colleagues (2010). Couples $(N=46)$ seen in a marriage and family therapy graduate training clinic that served the general community were randomized to a Feedback or TAU condition. Results of this study showed that couples using feedback achieved gains comparable to those found in the study by Anker colleagues' (2009). First, couples in the PCOMS condition of the current study experienced average gains of 8.30 ORS points (versus 8.58 in Anker et al., 2009). The ES between treatment conditions was also nearly identical ( $d=0.48$ versus $d=0.50)$. In addition, four times as many couples in the PCOMS condition achieved clinically significant change in both studies. Results of a multilevel growth model showed that couples in the PCOMS condition also improved in significantly fewer sessions than couples in the TAU condition.

Strengths and limitations. Strengths and limitations should be noted in relation to the client feedback literature using the OQ-45 system and PCOMS. First, the methodological rigor used to evaluate client feedback is excellent given that studies utilized the gold standard methodology of the RCT design with active control conditions (Lambert \& Ogles, 2004). Second, large samples were also generally used in the research, which is notable for naturalistic settings.

Some limitations are also of note. First, although the samples in the above studies were large, they were mostly comprised of university students and represented little ethnic diversity. In addition, more follow-up studies are needed to determine whether the effects of client feedback are maintained over time. Finally, most studies only used one 
outcome measure. Using additional outcome measures may enrich findings by broadening the definition of treatment outcome beyond global psychological functioning for a particular type of treatment and client population. For example, Anker et al. (2009) incorporated the Locke-Wallace Marital Adjustment Test (LWMAT; Locke \& Wallace, 1959) in their couples study to evaluate how treatment effected overall marital adjustment.

Overall, the research on the efficacy of client feedback demonstrates that client outcome is enhanced and retention is improved. Although the empirical evidence suggests that client feedback improves outcomes, questions remain. For example, why client feedback works is unclear. Sensibly, feedback improves outcomes and retention for clients who are NOT, but the research suggests that feedback is also beneficial for clients progressing as expected (although on-track clients using the OQ-45 System have smaller benefit). In addition, why client feedback improves retention for only clients who are NOT in a Feedback condition in two studies (Lambert et al., 2001a; Whipple et al., 2003) but for all clients in a Feedback condition in others (Lambert et al., 2002; Slade et al., 2008) is unclear.

Overall, client feedback has demonstrated impressive findings for individuals and couples in psychotherapy (Anker et al., 2009; Miller et al., 2006; Reese et al., 2009; 2010); however, still to be determined is whether client feedback enhances outcome with clients utilizing other formats of treatment such as group therapy. Three main rationales support studying the effectiveness of client feedback in group psychotherapy. First, applying a continuous assessment model to group therapy is worthy of study given its positive results for enhancing treatment outcome for both individuals (Reese et al., 2009; Shimokawa et al., 2010) and couples (Anker et al., 2009; Reese et al., 2010). Second, knowing that clients deteriorate and drop out at approximately equal rates across treatment 
formats (i.e., individual, couples, and group treatment) reinforces the need to monitor treatment outcome in groups routinely (Baekeland \& Lundwall, 1975; Lambert \& Ogles, 2004; Snyder, Castellani, \& Whisman, 2006; Wierzbicki \& Pekarik, 1993). Results from efficacy studies also suggest that client feedback may improve retention in therapy as well, particularly for clients who are NOT (Lambert et al., 2001a; 2002; Slade et al., 2008; Whipple et al., 2003). Finally, group treatment is becoming a popular treatment format due to its cost efficiency and demonstrated efficacy for treating a range of psychological concerns in a briefer period of time (Burlingame et al., 2003; Taylor et al., 2001). Given that feedback is considered a best practice for individual therapy (American Psychological Association [APA] Presidential Task Force, 2006), evaluating if such practice considerations should be extended to the group therapy format is warranted.

Client feedback in group therapy. Empirically, less is known about how client feedback contributes to group psychotherapy outcomes despite theoretical papers that posit its effectiveness (Dies \& Dies, 1993). Currently, only one published study has been conducted to evaluate whether client feedback in group psychotherapy contributes to improved outcomes. Davies, Burlingame, Johnson, Gleave, and Barlow (2008) studied the effects of a feedback intervention using the OQ-45 and Group Climate QuestionnaireShort version (GCQ-S; MacKenzie, 1983). The GCQ-S is comprised of 12 items designed to measure group members' perception of the climate across three domains: engagement (i.e., sense of closeness), avoidance (i.e., reluctance to accept responsibility for change), and conflict (i.e., presence of interpersonal friction). Feedback on the group climate was given in narrative and graphical (i.e., visual graph of GCQ-S subscale scores) forms to clients $(N=94)$, co-leaders, and the group-as-a-whole at a university counseling center every session. Researchers found that monitoring group climate did not enhance 
client outcome or level of engagement in the group compared to a TAU group. In fact, using the GCQ-S to obtain client feedback only had a statistically significant negative effect on outcome for clients who rated their group as having higher levels of conflict overall.

Several limitations of the study conducted by Davies and colleagues (2008) are of note. First, a high attrition rate was reported in this study. Clients also attended both individual and group therapy concurrently, which perhaps reduced the effect of the group and feedback intervention. Effects of the multifocal feedback were not controlled, which makes determining whether the focus of feedback influenced client outcome difficult. Finally, outcome was only monitored at pre and post therapy, thereby reducing the ability of the therapists to monitor client progress throughout the group. Still to be investigated is whether monitoring outcome routinely enhances client outcome and retention in group psychotherapy.

\section{Purpose of This Study}

The purpose of this study is to evaluate whether using client feedback enhances treatment outcome and retention in group psychotherapy. PCOMS was chosen as the client feedback system for this study given its brevity, cost (measures are free for use with license agreement), and familiarity, as the agency was already utilizing this measure in clinical practice for individual psychotherapy. In addition, a group alliance measure has already been developed and evaluated for use with the PCOMS system. Four hypotheses have been generated.

Hypothesis one. I hypothesize that statistically significant differences will be observed in pre-post psychotherapy outcome scores between group clients in a Feedback condition and group clients in a TAU condition. 
Hypothesis two. I hypothesize that statistically significant differences will be observed in the number of sessions needed to evidence statistically significant change in pre-post psychotherapy outcomes between group clients in a Feedback condition and group clients in a TAU control condition.

Hypothesis three. I hypothesize that statistically significant differences will be observed between group clients in the Feedback condition and group clients in a TAU condition with regards to percentages of reliable and clinically significant change.

Hypothesis four. I hypothesize that statistically significant differences will be observed between group clients in the Feedback condition and group clients in a TAU condition with regards to session attendance, drop out and deterioration rates, and premature termination.

\section{Summary}

Empirical evidence well supports the efficacy of group psychotherapy for the treatment of a variety of psychological concerns (Burlingame et al., 2003; Horne \& Rosenthal, 1997); however, research suggests that not all clients improve in psychotherapy or develop positive relationships with other members in the groups (Baekeland \& Lundwall, 1975; Norcross \& Wampold, 2011;Wierzbicki \& Pekarik, 1993). In addition, research supports that clinicians, on average, struggle to accurately identify clients who are at-risk of prematurely terminating treatment (Chapman et al., 2012; Hannan et al., 2005). Client feedback has demonstrated efficacy for improving premature termination (Lambert et al., 2001a; Whipple et al., 2003) as well as general psychotherapy outcome in both individual (Miller et al., 2006; Reese et al., 2009) and couples therapy (Anker et al., 2009; Reese et al., 2010) by monitoring client outcome and the alliance every session. 
Given the success yielded by client feedback in these formats, I will evaluate if the efficacy of client feedback extends to group psychotherapy using methods below.

Copyright (C) Norah Chapman Slone 2013 


\section{Chapter Two: Method}

The current study is an original data collection based on a cluster RCT design. Groups were randomly assigned to a Feedback group psychotherapy condition or a TAU group psychotherapy condition. Clients were assessed via self-report using PCOMS measures (ORS only in TAU condition; ORS and GSRS in Feedback condition) every session and another outcome measure at specified time points (pre and post and before every third session) throughout the study.

\section{Participants}

Research study participants $(N=85)$ were clients who participated in group therapy at a large university counseling center in the Southeast. A total of 44 clients participated in the Feedback condition and 41 clients participated in the TAU condition. Groups at the center are designed to last one academic semester. Clients were offered an average of 10 sessions (10.4 in the Feedback and 9.6 in the TAU condition) of group psychotherapy each semester. Two clients participated in both Fall and Spring 2012 semesters, each of them first participating in a TAU condition group then a Feedback condition group. All other members were new to the group therapy modality at this center and only attended one group for the intended duration.

\section{Group Leaders}

Graduate students, pre-doctoral interns, and doctoral level staff psychologists $(N=$ 20) served as group leaders. Two leaders were assigned to each group, one who had more group experience (e.g., senior staff member or experienced pre-doctoral intern), with a graduate student trainee in a clinical or counseling psychology doctoral program, pre-doctoral intern, or less experienced senior staff member. The Group Psychotherapy 
Coordinator, a licensed doctoral level staff psychologist at the counseling center, determined pairs of co-leaders at the outset of each semester prior to groups being randomized for the study and also provided weekly group supervision for approximately 1.5 hours with all group leaders. All group leaders received the same amount of supervision from the same supervisor throughout the duration of this study.

\section{Measures}

Consistent with procedures recommended in treatment outcome research (Lambert \& Ogles, 2004), two outcome measures were used in this study to evaluate research hypotheses and described below.

The Outcome Rating Scale (ORS; Miller \& Duncan, 2000). The ORS (see Appendix A) is a brief 4-item measure of global psychological functioning that was designed for use every session to monitor client outcome (Miller \& Duncan, 2004). Four domain scores derived from subscales of the OQ-45 are used to measure global psychological functioning using a visual analog scale $10 \mathrm{~cm}$ in length. Areas assessed include clients' functioning Individually (i.e., personal sense of well-being), Interpersonally (i.e., family, close relationships), Socially (i.e., work, school, friendships), and Overall (i.e., general sense of well-being). Although an electronic version of this measure exists, the paper-based version of the measure was utilized in this study. To administer this instrument, therapists ask clients to place a hash mark on the line that best represents how they were feeling the past week including that day. Scores on the right side of the scale indicate higher functioning. A centimeter ruler is used to score each domain to the nearest millimeter, with 10 being the highest score obtainable for each domain. A Total Score of 40 is possible on this measure. Once a Total Score is obtained, clients' scores are plotted on a graph (see Appendix C) for each session to track their 
progress throughout treatment. The ORS can be administered, scored, plotted, and interpreted in approximately one minute.

Two studies have provided empirical evidence for the validity and reliability of scores generated by the ORS (Bringhurst, Watson, Miller, \& Duncan, 2006; Miller, Duncan, Brown, Sparks, \& Claud, 2003). First, Miller and colleagues (2003) used both clinical and nonclinical samples to evaluate the psychometric properties of the ORS. A nonclinical sample of 86 master's students, therapists, and staff completed four consecutive administrations of the ORS across a span of 10 days -5 weeks. A proctor oversaw each of 336 total administrations. A staff receptionist obtained ORS data from a clinical sample $(N=435$ clients $)$ at pre and post treatment at a community family service agency. Results evaluating the psychometric properties of the ORS only incorporated data from the nonclinical sample. Internal consistency estimates using Cronbach's alpha was found to be .93 for all administrations (range of $.87-.97$ was noted from first to third administrations, respectively). The test-retest analysis yielded a correlation of $r$ $=.66$ at the second administrations but only $r=.49$ was found at the $4^{\text {th }}$ administration. This finding was significantly lower than the OQ-45 $(r=.74)$. Such scores are not surprising for brief outcome measures according to Miller and Duncan (2004). Second, Bringhurst and colleagues (2006) found similar results in a study conducted to replicate the findings of Miller et al. with a non-clinical sample of 98 participants recruited from a university. Estimates of internal consistency using Cronbach's alphas were similar to findings noted in Miller et al. (2003; .97 in this study, compared to .93) after four repeated administrations of the ORS across a 3-week period. Test-retest reliability estimates were higher in the replication study at $r=.80$ (compared to .66) after the second administration and $r=.81$ (compared to .58 ) after the third administration. These 
studies indicate that the ORS is an adequate measure for clinical use based on the above evidence that it generates reliable scores that are consistent over time. Internal consistency estimates for the current sample were high and within the ranges noted above at .92 across 327 total administrations. Test-retest reliability estimates were adequate and consistent with Miller et al. at .59 between sessions one and two.

Miller et al. (2003) and Bringhurst et al. (2006) also estimated concurrent validity in the studies noted above. Both studies utilized the OQ-45 as a measure of global psychological functioning to establish evidence of concurrent validity with the ORS. First, Miller and colleagues obtained a total of 335 paired administrations of the ORS and the OQ-45 across four sessions using a non-clinical sample $(N=86)$. Pearson product moment correlations revealed adequate evidence for concurrent validity with a strong relationship between the two measures on overall administrations $(r=.59)$. Second, Bringhurt and colleagues also replicated this finding and discovered a stronger relationship between the ORS and the OQ-45 $(r=.69)$ at the third administration (range of $r=.56-.57$ across first two administrations). Both studies revealed adequate evidence supporting concurrent validity of the ORS as a measure of global psychological functioning.

The Reliable Change Index (RCI; Jacobson \& Truax, 1991), a formula used to calculate whether the change in scores is larger than the standard error of measure, was developed to facilitate clinical use for the ORS to determine the amount of change needed to evidence reliable change. The RCI for the ORS was found to be 5 points (Miller et al., 2003). Miller and colleagues (2003) also found that the clinical cut off, or the differentiation in scores between clinical and nonclinical samples, for the ORS is 25 . To determine the clinical cut-off, Miller and colleagues compared mean ORS scores between 
a clinical sample from a community family service clinic $(n=435)$ and a non-clinical sample ( $n=86$; graduate students, therapists and staff noted above) across a minimum of three and a maximum of 10 administrations. A statistically significant difference was found between groups. A mean ORS score of approximately 25 at post-test for the clinical group was noted, meaning that clients who score below 25 are considered in the clinical range and present with distress at levels consistent with those who attend and benefit from therapy. Individuals who score above 25 are considered to be in the nonclinical range, meaning they are likely in less distress and typically show less improvement across therapy.

\section{Center Assessment of Psychological Symptoms - 34 (CCAPS-34; Locke et al.,} 2011). The CCAPS-34 (see Appendix E) is 34-item instrument used to assess the mental health of college students. The CCAPS-34 was normed on $N=9,560$ college students seeking counseling services from $N=97$ universities across the U.S. between 2010 2011. Administration of this measure can be completed via paper copy or on a computer module in 2-3 minutes. Both types of administration were used in this study for feasibility purposes. The measure uses a 5-point Likert-type scale ranging from 0 (not at all like me) to 4 (extremely like me) for each item. Total scores may range from $0-136$. There are seven subscales to measure the presence of mental health concerns: Depression, Generalized Anxiety, Social Anxiety, Academic Distress, Eating Concerns, Alcohol Use, and Hostility. In addition to these subscales, a Distress Index comprised of 20 items from across subscales was developed to monitor overall distress. Given that the purpose of this study is to monitor client distress over the course of treatment, only the Distress Index from this measure will be utilized in data analyses. 
A RCI (Jacobson \& Truax, 1991) was developed to denote the amount of change needed to be regarded as significant change on this measure and to facilitate clinical use to determine the amount of change needed to evidence reliable change. An RCI of .79 CCAPS-34 points was established for the Distress Index and used to determine whether reliable change (significant improvement or significant deterioration) was achieved during this study (Locke et al., 2012). A clinical cutoff was also generated for the CCAPS-34 based from two clinical cutoff scores and three ranges of severity and risk: Low, Mild, and Elevated. These scores are used to help clinicians determine whether a client's level of distress is clinically meaningful or below a level of clinical concern.

Evaluations of psychometric properties have only been published with the CCAPS64 (Locke et al., 2011, 2012). Internal consistency estimates are high, ranging from .84 .90 for Total and subscale scores. Test-retest estimates are also high ranging from .78 (Generalized Anxiety Scale) to .93 (Depression Scale) after one week and .76 (Academic Distress) to .92 (Depression) after two weeks. For the current sample, internal consistency estimates for the Distress Index were also high and within the range noted above (.89 at first administration; .93 at final administration). Test-retest reliability estimates for the Distress Index were evaluated at first and final administration (across approximately 3 weeks between $1^{\text {st }}$ and $2^{\text {nd }}$ administrations) and are also consistent with the range noted above at $r=.81$.

Evidence for convergent validity has been noted between domains of the CCAPS34 noted above and established measures including the Beck Depression Inventory (BDI; Beck, Ward, Mendelson, Mock, \& Erbaugh, 1961; $r=.72)$, Eating Attitudes Test-26 (EAT-26; Mintz \& O’Halloran, 2000; $r=.65)$, the Alcohol Use Disorders Identification Test (AUDIT; Saunders, Aasland, Babor, de la Fuente, \& Grant, 1993; $r=.81$ ), the Beck 
Anxiety Inventory (BAI; Beck, Epstein, Brown, \& Steer, 1988; $r=.64$ ), the Social Phobia Diagnostic Questionnaire (SPDQ; Newman, Kachin, Zuellig, Constantino, \& Cashman-McGrath, 2003; $r=.73$ ), Academic Adjustment (subscale of the Social Adjustment to College Questionnaire (SACQ; Baker \& Siryk, 1986; $r=-.68$ )), and the State-Trait Anger Expression Inventory-2 (STAXI-2; Spielberger, 1999; $r=.57$ ). For the current study's sample, evidence of convergent validity between the CCAPS-34 Distress Index and ORS Total score at session one was found to be strong $(r=.74)$.

\section{Procedures}

The efficacy of client feedback in group psychotherapy was evaluated utilizing a cluster RCT design to determine whether client feedback improves treatment outcome and retention in group psychotherapy compared to typical group therapy services, or group TAU, at a university counseling center. Randomization occurred at the group level in which groups were randomly assigned as a Feedback or TAU condition. Randomizing clients and co-leaders individually to groups was not feasible due to client, staff, and group leaders' schedules, as they were only available during certain times and therefore were offered group times based on availability.

Groups $(N=10)$ utilized in this naturalistic (i.e., university counseling center) setting were closed (i.e., more members were not added once formed), Yalom (1995) based Interpersonal Process psychotherapy (IP) groups conducted for an average of 10 sessions across two semesters (Spring and Fall 2012). Groups started over each semester with new members and co-leaders. Clients were asked to make a commitment to attend group therapy for the entire semester to achieve maximum benefits of the group experience. Each group was comprised of 8.5 clients on average (8.8 in Feedback condition $[S D=1.10]$; 8.2 in TAU condition $[S D=1.30]$ ). Group times and co- 
leadership assignments were made by the counseling center's Group Coordinator at the beginning of each semester and were not able to be managed by research personnel.

Clients who participated in group therapy at this center were recruited through referral procedures and inclusion/exclusion criteria outlined by center policies. Clients were referred to group therapy by their intake or individual therapist who presented with concerns related to social anxiety or other interpersonal issues, although some clients presented with other co-morbid concerns (i.e., depression, eating disorders) that were not controlled for in the analyses of this study, given the focus was on client feedback and not specific outcomes across diagnoses or conditions. Clients who were diagnosed with a personality disorder (those with traits or features of a personality disorder could be included), active substance abuse issues, acute suicidal ideation, autism spectrum disorders, developmental delays, intellectual disabilities, and/or psychosis were excluded from group therapy and referred for other counseling services offered by the center or local community agencies per policy of the counseling center.

In addition to procedures related to inclusion and exclusion criteria, several counseling center policies also contributed to participants' experiences in group therapy at this center. First, participants were not permitted to attend both individual and group therapy concurrently unless special circumstances warranted joint treatment. No one in this study was seen in both treatment formats, although three clients (one from a Feedback condition, two from a TAU condition) came in for a crisis walk-in (approximately a 30-minute problem solving appointment to assess risk level and provide coping strategies) at some point during the study. Two, the policy of the center and consistent with Yalom-based (1995) IP group practices is to include two leaders and a maximum number of 10 members in each group to ensure clients receive adequate time 
to work on their respective presenting concerns. Last, all groups at the center were digitally recorded for training purposes. Clients who did not wish to be recorded were unable to participate in group therapy at this center given that graduate student trainees are involved in the leadership of these groups and are required to have their clinical services digitally recorded for supervision and training purposes.

Informed consent. Prior to clients attending any psychotherapy services at this center, clients submitted their intake paperwork (including demographic information) electronically, which also contained an opt-in/opt-out statement (see Appendix D) about whether they consent to participate in research endeavors evaluating services at the center. Clients who endorsed participating in service evaluations had their data included in this study and research personnel excluded clients who opted out of the research study by filtering out their Student ID numbers upon data analysis.

Group screening. All clients referred to group therapy, regardless of condition, were provided with a group screening appointment prior to attending group therapy. Group screening appointments allowed clients to meet with group co-leaders to discuss group norms and expectations, establish clients' goals for group, and sign necessary paperwork (e.g., client confidentiality forms for group therapy, digital recording permission). Those interested and found to be appropriate for the group therapy program (according to inclusion/exclusion criteria outlines by the center and reviewed above) were provided with instructions about group attendance and information regarding the research study (see Appendices E and F for group screening script by condition). Clients who declined to participate in the study at the screening or on their intake paperwork were asked to respond to the measures to ensure they would not be affected (e.g., be 
singled out in the group) for not participating. Slight differences in the group screening procedures were delineated by condition and described below.

Feedback intervention protocol procedures. Research personnel provided training on the feedback intervention protocol to group leaders in the Feedback condition during group supervision prior to group screenings and group therapy sessions. Group leader demographic data were collected at that time. Leaders were provided approximately 1-hour of training on how to administer, score, plot, and interpret the ORS and GSRS. Ongoing support was provided to group leaders in the Feedback condition with regards to protocol concerns. During supervision, therapists provided research personnel with updates on logistical protocol issues only to avoid contaminating the control condition with specific questions regarding a formal check in with clients on their progress or the alliance. Any additional questions specific to obtaining and utilizing feedback with group members were communicated with research personnel on a more individualized basis.

Screening protocol. Group co-leaders provided instructions to their clients on how to respond to the ORS (Miller \& Duncan, 2000) and the CCAPS-34 (Locke et al., 2010) during their screening appointments. All clients who participated in the study were asked to write their Student ID numbers on top of their measures to link their pretreatment measures with subsequent assessments and to maintain confidentiality. Coleaders in the Feedback condition provided their group members with instructions on how to score and plot their ORS Total Scores on their individualized graphs identified by client initials during their screening appointment. Graphs were used throughout the duration of group therapy in a given semester to plot outcome scores to monitor client progress each session. Although these scores were not included in the data analysis, this 
process gave co-leaders and clients an opportunity to see the measures, score, and interpret the assessments in preparation for the first group session. This process was included in hopes that group members would be able to more quickly respond to these measures during the first group session and not take up more group time with research procedures. Clients were informed that procedures practiced in group screening will be routine for them during each session using the two measures associated with PCOMS for clients and therapists to have immediate feedback via their graphs each the session.

Group session protocol. Clients had an opportunity to discuss their progress according to their scores on the ORS and GSRS every session. At the beginning of each session, clients scored and plotted their own ORS Total Score on a graph that displays their treatment progress. Clients had an opportunity to address any reactions (e.g., noticed changes in their psychological functioning relative to previous week, particularly if below the clinical cutoff line of 25) they had concerning their individual progress in the group at that time (5 minutes or less). If clients did not offer reactions, group leaders encouraged group members to comment on their progress briefly to determine whether changes need to be made in the group to help improve their outcomes during that session. At the end of each group therapy session, clients responded to the GSRS, and they scored and plotted their Total Scores on their graph at that time. Again, leaders asked clients to share their reactions on their progress according to group alliance scores relative to their previous session score. Clients with scores below the cut off of 36 were asked to share what their needs were to improve their experience in the group.

Research personnel provided a "signal system" (see Appendix K) to all group coleaders in the Feedback condition after the third and each subsequent session. The signal system is a method that categorizes a client's progress on the ORS and GSRS from the 
previous week according to the PCOMS manual to efficiently inform co-leaders if their members are progressing as expected or at-risk for terminating treatment prematurely (Miller \& Duncan, 2004). For example, if a client declined by 10 points on the ORS from the session before, leaders would be alerted that their client is deteriorating, and it was recommended that their treatment progress and the alliance be explicitly reviewed with the client in the group next session. Prior to each group session, research personnel affixed index cards to client graphs with messages denoting members' progress based on categories developed by Miller and Duncan (2004). Each category provides a summary of how each client has changed from the previous week with recommendations for how to intervene during the next group session described below:

- No change. Clients who have not made at least a 5-point increase or decrease in the first three sessions are at increased risk of treatment failure, or premature termination from therapy. Leaders were directed to address the deterioration and inquire about clients' perspectives of therapy and the alliance. If clients remain unchanged after three additional sessions (session six), leaders had a discussion with clients about their perception of the group alliance, whether the treatment format is beneficial, or determine whether a medication consultation is necessary. This type of progress along with recommended interventions was indicated to group leaders on a yellow card.

- Reliably deteriorated. Clients who have deteriorated by at least 5-points from their baseline measure are reliably deteriorated and considered to be at risk for dropping out of treatment prematurely. Leaders were directed to address the deterioration and inquire about clients' perspectives of therapy and the alliance. If clients continued to deteriorate, leaders spoke with clients about 
alternative treatment options and possible medication referrals. This type of progress along with recommended interventions above were indicated to group leaders on a red card.

- Reliably changed. Clients who have made at least a 5-point improvement on the ORS have experienced reliable change. Leaders with clients in this range reinforced progress and specific changes clients made and assisted clients with further refining their "change strategies." This type of progress along with recommended interventions was indicated to group leaders on a blue card.

- Clinically significant change. Clients who have experienced a 5-point gain that crossed the clinical cutoff of 25 on the ORS have made clinically significant and reliable change, meaning clients are experiencing recovery. Leaders assisted clients in this category by refining their "change strategies" and focused on preparing them for potential relapses or setbacks. This type of progress along with recommended interventions was indicated to group leaders on a green card.

Co-leaders were instructed to talk with one another about how to best address and incorporate this feedback in the group weekly using general guidelines provided by the PCOMS manual and described above (Miller \& Duncan, 2004).

Treatment fidelity was monitored through research personnel's attendance in weekly group therapy supervision. During supervision meetings, co-leaders verbally confirmed that they were checking in with clients about their scores on the ORS and GSRS each session and discussing their clients' feedback scores and progress with one another prior to each group session. This method of monitoring treatment adherence is 
consistent with one form of treatment fidelity well known in the literature (Hogue, Liddle, \& Rowe, 1996).

TAU condition protocol procedures. Research personnel provided training on the TAU protocol during group supervision prior to group screenings and group therapy sessions each semester. Group leader demographic data were collected at that time. Leaders were provided approximately 1 -hour of training on how to administer the ORS and CCAPS-34. Ongoing support was provided to group leaders with regards to protocol concerns. During supervision, group leaders provided research personnel with updates on logistical protocol issues.

Screening protocol. Group co-leaders assigned to the TAU condition provided instructions to their clients on how to respond to the ORS (Miller \& Duncan, 2000) and the CCAPS-34 (Locke et al., 2011) during their screening appointments; however, clients nor co-leaders scored these measures. Again, all clients who participated in the study were asked to write their Student ID number on top of their measures to link their pretreatment measures with subsequent assessments and to maintain confidentiality. Coleaders in the TAU condition simply returned un-scored ORS and CCAPS-34 measures to research personnel to score at another time.

Group session protocol. Clients completed the ORS immediately before each group session. Measures were simply handed in to leaders at the end of each session and scored by research personnel at another time. Co-leaders did not have access to their clients' progress throughout the duration of the research study. Leaders were instructed not to prompt any discussions on client progress as related to the measures.

CCAPS-34. One additional outcome measure was administered to all participants to evaluate the measures' convergence with the ORS as well as add specificity to the 
understanding of group psychotherapy outcome across domains noted on this measure. Clients in the Feedback and TAU conditions completed the CCAPS-34 measure at the beginning of the first, final, and every three sessions in between. The CCAPS-34 results were not discussed in the Feedback or TAU condition, nor did leaders have access to client progress via this measure.

Special considerations. Several special considerations were noted in the protocol for logistical and research purposes. First, when clients arrived late to the group therapy session, they responded to the ORS (and CCAPS-34 if given that week) at the end of group so as not to disturb the group process upon their arrival. Clients in the Feedback condition who missed a discussion of their ORS at the beginning of the group due to tardiness responded to and plotted their scores at the end of the group to alert co-leaders of their progress. If clients missed a week of therapy, they had missing session data for that week. Clients who missed a week of therapy in which the CCAPS-34 was administered were asked to respond the next session they attended. Alterations to the research protocol for special considerations were noted for research personnel.

\section{Data Analyses}

Statistical analyses were conducted to evaluate the research hypotheses using HLM 7.0 (Raudenbush, Bryk, \& Congdon, 2004) and IBM SPSS 20.0 (IBM, 2011). Descriptive statistics (i.e., means, standard deviations, ranges) were calculated for all study measures by treatment condition and study sample characteristics (i.e., mean age, percentages for ethnic and gender identity categories, and academic classification). To determine whether treatment randomization was successful, independent samples $t$-tests were calculated to compare group sample sizes and mean pre-treatment ORS scores across treatment conditions. Standardized ES's, Cohen's $d$, (Cohen, 1988), were also calculated 
to quantify the magnitude of the effect noted by Feedback across the Feedback and TAU conditions on outcome measures (Coe, 2002). The calculation for ES size within groups was: $M_{\text {post }}-M_{\text {pre }} / S D_{\text {pre. }}$ The calculation used to determine the ES of Feedback, or a between group ES, at post-test was: $B_{01} / \sqrt{ }\left(\left[n_{\mathrm{TAU}}-1\right] s^{2}\right.$ TAU Post-ORS $+\left[n_{\text {Feedack }}-1\right] s^{2}$ Feedback PostORS $/ N-2$ ) Feedback Post-ORS, which is the same formula used in previous PCOMS studies evaluating client feedback efficacy with couples (Reese et al., 2010). Interpretation of the ES according to Cohen $(1980,1988)$ is described in the following way: An effect is small if the ES is at least $d=0.20$; medium if the ES is $d=0.50$, and large effect if $d=0.80$ or larger. Inferential statistics varied across hypotheses and are described in detail below.

Hypotheses one. I hypothesized that statistically significant differences would be observed in pre-post psychotherapy outcome scores between group clients in a Feedback condition and group clients in a TAU condition.

$$
\begin{aligned}
& H_{1}: \beta_{\text {Feedback }} \neq \beta_{\mathrm{TAU}} \\
& H_{0}: \beta_{\text {Feedback }}=\beta_{\mathrm{TAU}}, \\
& H_{1}: d_{\text {Feedback }} \neq d_{\mathrm{TAU}} \\
& H_{0}: d_{\text {Feedback }}=d_{\mathrm{TAU}}
\end{aligned}
$$

where Feedback is operationalized as the presence of monitoring client outcome in therapy using two brief assessments of their outcome and alliance every session. Outcome is defined as clients' post-treatment score on the ORS and CCAPS-34. Although the ORS and GSRS were implemented together for the Feedback intervention, the GSRS was not used in the analyses but rather was part of the intervention.

Given the nature of the data was collected at the group level, a two level multilevel model (Hox, 2002) was initially selected as the primary statistical analysis to account for the interdependent, or the nested, nature of group level data (i.e., repeated measures, 
clients within groups; Raudenbush \& Bryk, 2002). Accounting for the nested variables reduces Type I error and acknowledges that group members may respond more similarly to one another within groups than between groups (Tasca, Illing, Joyce, \& Ogrodniczuk, 2009). Similar models and data analysis were used to account for nested data in a study using client feedback with couples and when evaluating general group psychotherapy outcome (see Reese et al., 2010; Tasca et al., 2009). Tasca and colleagues (2009) suggested that two primary assumptions be met before using multilevel models in group psychotherapy research: (a) data are nested within a hierarchical structure, and (b) the amount of variance in change is related to the nested structure of the data. An estimate of variance attributed to the nested structure was calculated using an Intraclass Correlation Coefficient (ICC) using the random variance component at the highest level of the model/the sum of random variance components at each level, which in this study is as follows: $\partial^{2}$ group $/ \partial^{2}$ client $+\partial^{2}$ group. When calculating the ICC for this study data, a two level unconditional model was constructed to calculate variance estimations attributed to each level of analysis. An ICC was calculated from variance estimates from the unconditional model to determine whether data met assumptions for dependence prior to proceeding with HLM procedures. The ICC can be calculated using the following formula: $\mathrm{ICC}=$

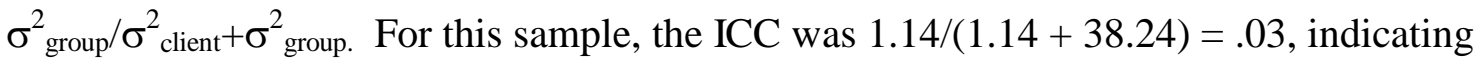
that approximately $3 \%$ of the variance is explained by between group differences when the initial ORS score is controlled. Given the ICC was low, analyzing data using HLM procedures was not necessary (Raudenbush \& Bryk, 2002), as it indicates that are no unique variance estimates attributable to group level data. Therefore, univariate statistical methods of linear regression were employed to evaluate data to address hypothesis one. 
A Generalized Linear Model was chosen as the primary method of statistical analysis used to evaluate the efficacy of client feedback. It was utilized to accommodate approaches for response variables as well as flexibility with regards to evaluating nonnormal data (Seiss, 2010). Methods for analyzing data according to this procedure included first checking the assumptions needed for this type of analysis, particularly normality as well as homoscedasticity, or the assumption that the error terms have a constant variance (McCue et al., 2008). The model response variable (Post-test ORS; continuous variable), a covariate (Pre-test ORS scores; continuous), and a dichotomous predictor (Feedback; categorical, coded $0=$ TAU condition, $1=$ Feedback condition) were used to evaluate this first hypothesis. Two models were constructed: (a) A covariate only model to control for initial treatment functioning; (b) Conditional Model in which the Feedback variable was entered to evaluate whether outcome differed statistically significantly across treatment conditions.

Two primary methods were used to evaluate both model parameters and goodness of fit. First, model parameters were evaluated using a maximum likelihood estimation technique, or method of estimating parameters in which the probability distribution is considered "most likely" given the observed data, as is standard when using a Generalized Linear Model. A benefit to this type of estimation technique is the flexibility with regards to data normality and linearity (Myung, 2002). Second, evaluating model fit to the data was conducted using a -2 Log Likelihood (-2LL) deviance statistic, to evaluate if the Conditional Model was comprised of parameters that fit the data in such a way that were statistically significantly different from zero, indicated by an Omnibus test in SPSS.

Hypothesis two. I hypothesized that statistically significant differences will be observed in the number of sessions needed to evidence statistically significant change in 
pre-post psychotherapy outcomes between group clients in a Feedback condition and group clients in a TAU control condition.

$$
\begin{aligned}
& H_{1}: \beta_{\text {Feedback growth }} \neq \beta \text { TAU growth } \\
& H_{0}: \beta_{\text {Feedback growth }}=\beta \text { TAU growth, }
\end{aligned}
$$

where, again, Feedback is operationalized as the presence of monitoring client outcome in therapy using two brief assessments of their outcome and alliance every session. Outcome is defined as clients' post-treatment score on the ORS and CCAPS-34. Sessions is operationalized as the number of sessions attended by clients in each condition. Growth is operationalized as linear, quadratic, and cubic rates by which clients improve across the duration of treatment on the ORS. Again, although the ORS and GSRS were implemented together for the Feedback intervention, the GSRS was not used in the analyses, but was part of the intervention.

A two-level multilevel model (Hox, 2002) was selected as the primary statistical analysis to evaluate hypothesis two to account for the interdependent, or the nested, type of group level data collected with repeated measures (i.e., repeated measures, clients within groups; Raudenbush \& Bryk, 2002). As mentioned above, Tasca and colleagues (2009) suggested that two primary assumptions be met before using multilevel models in group psychotherapy research: (a) data are nested within a hierarchical structure, and (b) the amount of variance in change is related to the nested structure of the data. An estimate of variance attributed to the nested structure was calculated using an ICC using the random variance component at the highest level of the model/the sum of random variance components at each level, or $\partial^{2}$ group $/ \partial^{2}$ client $+\partial^{2}$ group. When calculating the ICC for this study, a two-level unconditional model was constructed to calculate variance estimations attributed to each level of analysis. An ICC was calculated from variance estimates from 
the unconditional model to determine whether data met assumptions for dependence prior to proceeding with HLM procedures. The ICC can be calculated using the following formula: ICC $=\sigma_{\text {group }}^{2} / \sigma^{2}$ client $+\sigma^{2}$ group. For this sample, the ICC was $32.97 /(32.87+33.17)$ $=.50$, indicating that approximately $50 \%$ of the variance in rate of growth is explained by between group differences (or differences at Level 2) when initial ORS scores are controlled. Given this, the large ICC, using a multilevel growth model is appropriate.

A two-level growth model (repeated measures nested within groups) was constructed to evaluate if the rate of client improvement is different for group members in the Feedback condition compared to members in the TAU condition on the ORS. Using a growth curve model takes into account non-linear patterns that represent changes in ORS scores without assuming this change is linear (Aycock, n.d.; Tasca et al., 2009). The CCAPS-34 was not used in this analysis given it was administered less routinely throughout the study and may not capture session-to-session change as precisely.

Post-test ORS scores served as the criterion variable, or the clients' final observed ORS score. Pre-treatment ORS scores were centered on 0 to control for client pretreatment differences in the growth model. Session number (a fixed continuous variable) was added at Level 1 as well as a Session ${ }^{2}$ quadratic term (fixed continuous variable) to capture linear and non-linear growth across and between sessions. At Level 2 (group level), a quadratic (Feedback*Session; fixed continuous variable) and a cubic term $($ Feedback $)\left(\right.$ Session $\left.^{2}\right)$ were entered to determine whether differences in non-linear growth rate between groups significantly differed across treatment condition. Additional analyses were conducted to evaluate how well the Conditional Model fit the data in comparison to the Unconditional, or null, Model. Parameter estimates were evaluated using a restricted 
maximum likelihood estimation method. The Chi-square statistic was used to compare the deviance statistic (-2LL) from the Covariate Only Model to the Conditional Model.

Hypothesis three. I hypothesized clients in the Feedback condition would achieve statistically significant higher rates of reliable and clinically significant change than those in the TAU condition on the ORS and CCAPS-34.

$$
\begin{aligned}
& H_{1}: X^{2} \text { Feedback reliable change } \neq X^{2}{ }_{\text {TAU reliable change }} \\
& H_{0}: X^{2}{ }_{\text {Feedback reliable change }}=X^{2}{ }_{\text {TAU reliable change, }} \\
& H_{1}: X^{2} \text { Feedback clinically significant change } \neq X^{2}{ }_{\text {TAU clinically significant change }} \\
& H_{0}: X^{2}{ }_{\text {Feedback clinically significant change }}=X^{2}{ }_{\text {TAUclinically significant change }}
\end{aligned}
$$

where reliable change (categorical) is operationalized as a gain of 5 points from pre to post group therapy on the ORS and clinically significant change (categorical) is defined as an improvement of at least 5 points on the ORS and a change in score that crosses the clinical cutoff or 25 from pre to post group therapy.

Descriptive statistics were used to calculate the percentage of clients across conditions who achieved reliable change (5-point improvement on ORS scores from pre to post), clinically significant change (those in the clinical range initially who achieve scores of at least 5 or more ORS points at post-test in the non-clinical range of functioning), reliable deterioration (5 point reduction on ORS scores from pre to post test), or no change (change from pre to post test on the ORS is within 5 points, or not greater than the standard error of measure on this instrument). In addition, the RCI for the CCAPS-34 Distress Index was used to determine whether client changes from pre to post were classified as Low (below a mean score of 1.21), Mild (a mean CCAPS-34 Distress Index score of between 1.22 and 2.15), or Elevated (a mean CCAPS-34 Distress Index score above 2.15) to determine degree of change from pre to post test on the 
measure. Chi-square analyses were used to determine whether statistically significant differences in the number of clients who met criteria for each category were noted between Feedback and TAU conditions on each of the outcome measures.

Hypothesis four. Finally, I hypothesize that clients in the Feedback condition will attend more sessions overall, drop out and deteriorate at a lower rate, and fewer will terminate prematurely from therapy compared to clients in the TAU condition.

$$
\begin{aligned}
& H_{1}: t_{\text {Feedback session number }} \neq t \text { TAU session number } \\
& H_{0}: t_{\text {Feedback session number }}=t \text { TAU session number, } \\
& H_{1}: X^{2} \text { Feedback deterioration } \neq X^{2} \text { TAU deterioration } \\
& H_{0}: X^{2} \text { Feedback deterioration }=X^{2} \text { TAU deterioration, } \\
& H_{1}: X^{2} \text { Feedback dropout } \neq X^{2}{ }_{\text {TAU dropout }} \\
& H_{0}: X^{2} \text { Feedback dropout }=X^{2}{ }_{\text {TAU dropout }} \\
& H_{1}: N_{\text {Feedback premature termination }} \neq N \text { TAU premature termination } \\
& H_{0}: N_{\text {Feedback premature termination }}=N_{\text {TAU premature termination, }}
\end{aligned}
$$

where session was operationally defined as the number of sessions attended (continuous) relative to the number of sessions provided (continuous). Again, deterioration was operationalized as scores (continuous) from pre to post test that were $\geq 5$ ORS points lower from baseline, or session 1. Drop out was operationalized as clients who did not attend at least $75 \%$ of group treatment sessions (categorical; coded for completion, $1=$ yes, $0=$ no). As mentioned above, premature termination was operationalized as clients who dropped out of group therapy (categorical, coded for completion, $1=$ yes, $0=$ no) before reaching the clinical cutoff of 25 on the ORS (categorical; coded for indication of premature termination criteria, $1=$ yes, $0=$ no). Independent samples $t$-tests and Chisquare analyses were computed to determine whether attendance, deterioration, drop out, 
and premature termination rates across treatment conditions were statistically significantly different and whether any differences on initial functioning and demographic variables on the ORS and CCAPS-34 were noted between treatment completers and those who dropped out of therapy.

\section{Summary}

In sum, methods to evaluate the efficacy of client feedback in group psychotherapy were supported by a RCT design in a university counseling center setting. Groups were randomized to a Feedback or TAU group psychotherapy condition in which clients from this center were either provided feedback to their group leaders on their perceptions of outcome and the group alliance each session or received TAU. Group leaders in the Feedback condition were charged with utilizing client feedback provided via the ORS and GSRS each session as well as incorporating information from the signal system after session 3 to more accurately identify clients who were at-risk of dropping out, deteriorating, and/or prematurely terminating treatment. Methods described were carried out to evaluate research hypotheses and results are described below. 


\section{Chapter Three: Results}

Data analysis was based on $N=85$ clients who attended at least two group therapy treatment sessions from January 2012 to December 2012. A total of $N=90$ clients began group therapy; however, three clients only attended one session before dropping out and two clients opted out of the research study while doing intake paperwork and were removed from analyses. A total of 23 clients had at least two data points that could be used in the analysis; however, they dropped out of the study and data imputations for final post test scores were comprised of their last observations carried forward to account for their post-test ORS score $(\mathrm{Xu}, 2009)$, as it often used in longitudinal psychotherapy outcome studies in naturalistic settings (See Shimokawa, Lambert, \& Smart, 2010; Slade et al., 2008). Additional analyses around clients who dropped out of the study will be addressed below given it is part of research hypothesis four.

Client participants had a mean age of $21.50(S D=2.69$; range $18-28)$, which was nearly identical across treatment conditions (mean age in Feedback condition: 21.57, mean age in TAU condition 21.50$)$ and identified as mostly women $(63.95 \% ; 36.05 \%$ identified as men), Caucasian (87.21\%; $12.79 \%$ identified as African American/Black, $1.16 \%$ as Multiracial, and $1.16 \%$ asked to Self-Identify). They described their sexual identity as mostly heterosexual (86.05\%), though some clients identified as gay (4.65\%), lesbian (1.16\%), bisexual (3.49\%), or questioning (1.16\%). Clients also described their relationship status as being mostly single (61.63\%), though some were in a serious or dating or committed relationship (29.07\%), married (3.49\%), or divorced (2.33\%).

Clients who comprised the sample of this study were Freshmen $(23.26 \%)$, Sophomores (13.95\%), Juniors (23.26\%), Seniors (36.59\%), and Graduate or Professional Degree 
students (22.09\%). Although clients' specific presenting concerns or diagnoses were not monitored in this study, clients at this center endorsed the following top 10 presenting concerns in order of highest percentage endorsed (more than one could be checked): Anxiety (68\%), Stress (64\%), Depression (58\%), Academics (44\%), Concentration (42\%), Relationships (38\%), Self-esteem (36\%), Family (34\%), Loneliness (34\%), Time management/motivation (31\%). A clear majority of clients in this sample received individual psychotherapy prior to beginning group therapy (56.98\%; $43.02 \%$ had not). The recency of their psychotherapy experience was not available. The use of psychotropic medication was also not monitored in this study. There were no significant differences noted on demographic variables across treatment conditions $(p>.05)$. Please see Table 3.1 for a full breakdown of demographic variables by treatment condition.

Group leaders were a mean age of $32.1(S D=8.2$; range of 23-52), mostly identified as women (80\%; $20 \%$ identified as men) and identified as Caucasian (85.0\%), Asian/Pacific Islander (10.0\%), and Multiracial (5.0\%). Eight group leaders had doctoral degrees, and 12 were doctoral student trainees (eight had a master's degree in a psychology related field, two of which were pre-doctoral interns, and four had a bachelor's degree). Group leaders reported an average of 4.75 years of group psychotherapy experience ( $S D=7.10$; range $0-22$ years) and had led an average of 4.5 groups $(S D=4.40$; range: $0-15)$ prior to this study. They identified their general theoretical orientations as mostly integrative $(65.0 \%)$, though some identified primarily with Interpersonal Process Therapy (20.0\%) and Cognitive Behavioral Therapy (15.0\%) orientations. No differences were noted across treatment conditions on any group leader demographic variables. 
Table 3.1

Demographic Data for Client Participants $(N=85)$

Feedback condition TAU condition

$$
(n=44) \quad(n=41)
$$

Gender

Man

$\begin{array}{llll}16 & 35.56 & 15 & 36.59\end{array}$

Woman

29

64.44

26

63.41

Ethnicity

Caucasian

African American/Black

Multiracial

Self-identify

Classification

Freshman/First year

Sophomore

Junior

Senior

Graduate/Professional degree
38

4

1

1

2.22

8.89

2.22

35

5

12.20

10

22.2

10

5

11.11

7

17.07

14

31.11

6

14.63

8

17.78

7

17.07

8

17.78

11

26.83

Relationship Status

Single

28

62.22

25

60.98

Table 3.1 continues 
Table 3.1 continued

Feedback condition TAU condition

$$
(n=44) \quad(n=41)
$$

\begin{tabular}{|c|c|c|c|c|}
\hline & $N$ & $\%$ & $N$ & $\%$ \\
\hline Serious dating or & 12 & 26.67 & 13 & 31.71 \\
\hline \multicolumn{5}{|c|}{ committed relationship } \\
\hline Married & 2 & 4.44 & 1 & 2.44 \\
\hline Divorced & 2 & 4.44 & & \\
\hline \multicolumn{5}{|l|}{ Sexual Identity } \\
\hline Heterosexual & 39 & 6.67 & 35 & 85.37 \\
\hline Gay & 3 & 6.67 & 1 & 2.44 \\
\hline Lesbian & 2 & 4.44 & 1 & 2.44 \\
\hline Bisexual & & & 3 & 7.32 \\
\hline Questioning & 1 & 2.22 & 1 & 2.44 \\
\hline \multicolumn{5}{|l|}{ Previous Counseling } \\
\hline Yes & 27 & 60.00 & 22 & 53.67 \\
\hline No & 18 & 40.00 & 19 & 46.34 \\
\hline
\end{tabular}

Note. $\mathrm{TAU}=$ Treatment as Usual. 


\section{Preliminary Analysis}

To evaluate if randomization across groups was successful, pre-treatment ORS and CCAPS-34 were compared. No statistically significant differences were noted between the two treatment conditions for the ORS $(t(83)=0.83, p>.05)$ or CCAPS-34

Distress Index, $t(81)=0.81, p>.05$, gender $\left(\chi^{2}(2, N=85)=0.58, p>.05\right)$, or race/ethnicity $\left(\chi^{2}(2, N=85)=0.72, p>.05\right)$, offering support that initial levels of distress were equivalent across groups and randomization was successful.

\section{Results of Hypotheses}

The follow describes the results of statistical analyses utilized to evaluate research hypotheses.

Hypothesis one. I hypothesized that statistically significant differences would be observed in pre-post psychotherapy outcome scores between group clients in a Feedback condition and group clients in a TAU condition on the ORS and CCAPS-34. The average amount of change from pre to post on the ORS was 7.04 points $(d=0.90), 95 \% \mathrm{CI} \pm .37$

$[0.55,1.29]$ within the Feedback condition and 5.24 points $(d=0.74), 95 \% \mathrm{CI} \pm .35[0.37$, 1.02] within the TAU condition. These within group ES's can be considered large by Cohen's (1988) standards. Results of the ES between conditions, and an effect of Feedback, was $d=0.35$ on the ORS when initial functioning is controlled $(d=.44$ when initial functioning was not controlled), which is a small to medium ES by Cohen's (1988) standards. Clients experienced a reduction in scores on the CCAPS-34 Distress Index of 0.16 CCAPS-34 points $(d=0.24)$ in the Feedback condition and -0.26 CCAPS-34 Distress Index points in the TAU condition $(d=0.33)$. Both ES's were small to medium according to Cohen's (1988) standards. Please see Table 3.2 for means, standard deviations, and ES for both treatment conditions and the total sample. 
Table 3.2

Means, SD, and ES's for ORS and CCAPS-34

\begin{tabular}{|c|c|c|c|c|c|c|}
\hline & \multicolumn{2}{|c|}{$\begin{array}{c}\text { Feedback } \\
\text { condition } \\
(n=44)\end{array}$} & \multicolumn{2}{|c|}{$\begin{array}{c}\text { TAU } \\
\text { condition } \\
(n=41)\end{array}$} & \multicolumn{2}{|c|}{$\begin{array}{c}\text { Total } \\
(N=85)\end{array}$} \\
\hline & $M$ & $S D$ & $M$ & $S D$ & $M$ & $S D$ \\
\hline Pre-ORS & 23.36 & 7.80 & 22.02 & 7.06 & 22.71 & 7.44 \\
\hline Post-ORS & 30.40 & 7.15 & 27.26 & 6.85 & 28.88 & 7.14 \\
\hline \multirow[t]{2}{*}{$\operatorname{ES}(d)$} & \multicolumn{2}{|c|}{0.90} & \multicolumn{2}{|c|}{0.74} & \multicolumn{2}{|c|}{0.83} \\
\hline & \multicolumn{2}{|c|}{$\begin{array}{c}\text { Feedback } \\
\text { condition } \\
(n=40)\end{array}$} & \multicolumn{2}{|c|}{$\begin{array}{c}\text { TAU } \\
\text { condition } \\
(n=36)\end{array}$} & \multicolumn{2}{|c|}{$\begin{array}{c}\text { Total } \\
(N=76)\end{array}$} \\
\hline Pre-CCAPS-34 & 1.62 & 0.66 & 1.60 & 0.79 & 1.59 & 0.70 \\
\hline Post CCAPS-34 & 1.46 & 0.81 & 1.34 & 0.81 & 1.39 & 0.80 \\
\hline $\mathrm{ES}(d)$ & \multicolumn{2}{|c|}{-0.24} & \multicolumn{2}{|c|}{-0.33} & \multicolumn{2}{|c|}{-0.26} \\
\hline
\end{tabular}

Note. TAU $=$ Treatment As Usual. $M=$ Mean. $S D=$ Standard Deviation.

Pre-ORS = Pre-treatment functioning on the Outcome Rating Scale. Post-

ORS $=$ Post-treatment functioning on the ORS. ES $=$ Effect size. $d=$

Cohen's measure of sample effect size. Pre-CCAPS-34 = Pre-treatment

functioning on the Counseling Center Assessment of Psychological

Symptoms-34. Post-CCAPS-34 = Post-treatment functioning on the

Counseling Center Assessment of Psychological Symptoms-34. 
I first assessed the distribution and homoscedasticity of the dependent variable (Post-test ORS). A test of normality using the Shapiro-Wilks test was computed given the small sample size, which yielded statistically significant results $t(85)=.951, p=0.003$. Such results indicate that the response variable was non-normally distributed. In addition, there is an uneven scatter of Post-test ORS scores across the sample, indicating that heteroscedasticity is present. The Generalized Linear Model is an appropriate test to utilize, particularly evaluating the parameters with robust standard errors to account for a non-normal distribution as well as heteroscedasticity.

An evaluation using the Generalized Linear Model yielded statistically significant results for the covariate (Pre-test ORS; $\beta=.454, p<0.05)$ and Feedback predictor $(\beta=-$ 2.52, $p=0.05)$. Results suggest that when client's initial functioning is controlled, those in the Feedback condition are predicted to have treatment gain on average of $2.54 \mathrm{ORS}$ points from pre to post group therapy. This analysis yielded statistically significant results, suggesting that clients in the Feedback condition are predicted to yield statistically significantly improved pre-post gains in group therapy (See Table 3.3). In addition, an evaluation of model fit using the Omnibus test revealed that the covariate and Feedback predictor statistically significantly improved the model fit compared to an intercept-only model $\left(X^{2}[2, N=85]=26.84, p<.05\right)$.

A similar analysis was conducted to evaluate pre-post gains on the CCAPS-34. An evaluation using the Generalized Linear Model yielded statistically significant results for the covariate (Pre-test CCAPS-34; $\beta=.844, p<0.001$ ); however, the Feedback predictor was not statistically significant $(ß=-.105, p=.380)$. Results suggest that when client initial functioning is controlled, those in the Feedback condition are predicted to 
Table 3.3

Results of Generalized Linear Model Predicting Post Group Treatment ORS

Scores $(N=85)$

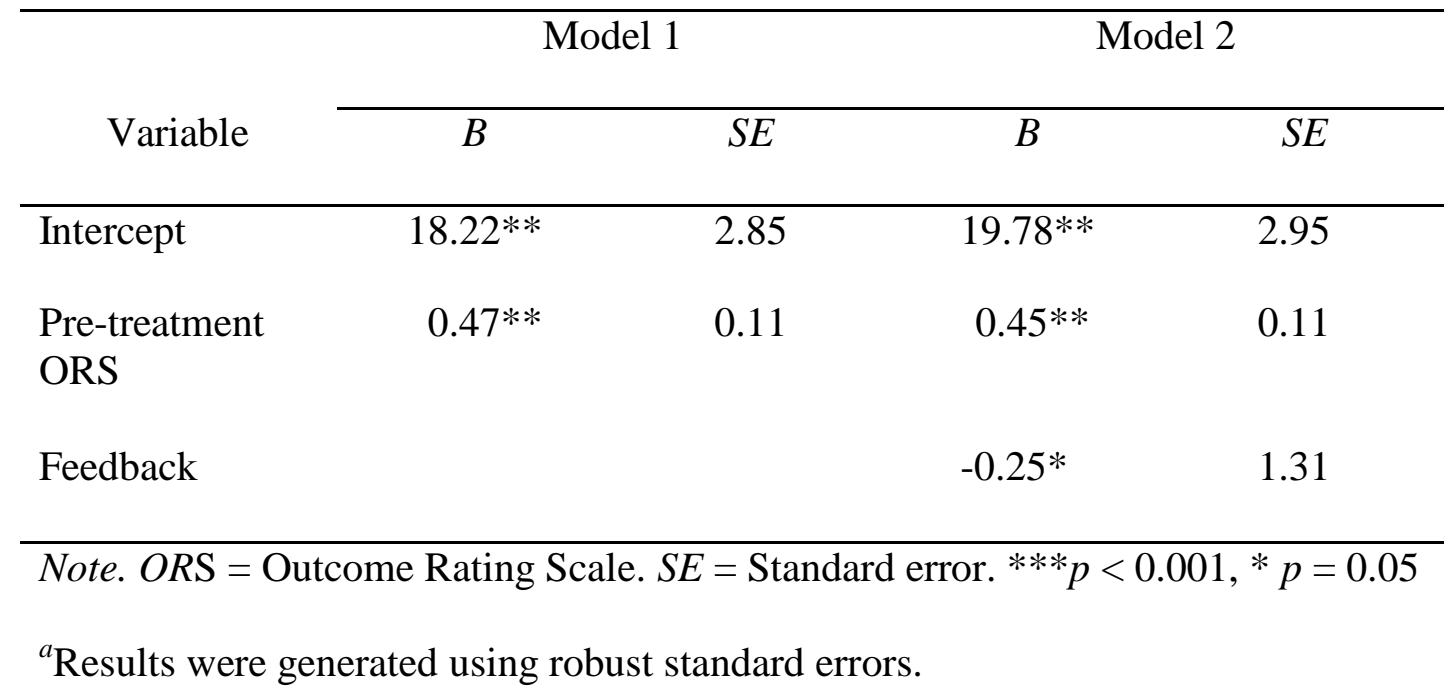


experience an average of 0.105 CCAPS-34 points improvement from pre to post group therapy; however, this degree of difference did not reach statistical significance (See Table 3.4). Although Feedback did not reach statistical significance, an evaluation of the fit of the statistical model revealed a statistically significantly improved model compared to an intercept only model $\left(X^{2}[2, N=76]=64.68, p<.05\right)$, indicating that controlling for the initial functioning of clients in the sample as a whole provided a model that significantly improved the fit to the data. Results from the regression analyses and descriptive statistics evidenced support for hypothesis 1.

Results yielded from a Generalized Linear Model analysis evidenced that clients in a Feedback condition achieved statistically significant more change when compared to the TAU condition on the ORS. These results were not replicated with the CCAPS-34 Distress Index scores however. Conclusions made from the analyses of the ORS are similar to previous research (Anker et al., 2009; Reese et al., 2009, 2010). Although evidence for the efficacy of client feedback was positive, ES's when controlling for initial functioning were lower than expected compared to previous PCOMS research.

Hypothesis two. I hypothesized that statistically significant differences will be observed in the number of sessions needed to evidence statistically significant change in pre-post psychotherapy outcomes between group clients in a Feedback condition and group clients in a TAU control condition. A two-level growth model (repeated measures, or sessions, nested within groups) was constructed to evaluate if the rate of change is statistically significantly different between group members in the Feedback condition compared to members in the TAU condition.

As recommended by Tasca and colleagues (2009), a base model, or an Unconditional Growth Model was first constructed to estimate variance at each level of 
Table 3.4

Results of Generalized Linear Model Predicting Post Group Treatment

CCAPS-34 Distress Index Scores $(N=76)$

\begin{tabular}{lcccc}
\hline & \multicolumn{2}{c}{ Model 1 } & \multicolumn{2}{c}{ Model 2 } \\
\cline { 2 - 5 } \multicolumn{1}{c}{ Variable } & $B^{a}$ & $S E$ & $B^{a}$ & $S E$ \\
\hline Intercept & $32.42^{* *}$ & 2.11 & $33.88^{* *}$ & 2.29 \\
CCAPS-34 & -0.22 & 1.28 & -2.22 & 0.11 \\
$\begin{array}{l}\text { Distress } \\
\text { Index 1 }\end{array}$ & & & & \\
Feedback & & & -0.30 & 1.50 \\
\hline
\end{tabular}

Note. CCAPS-34=Counseling Center Assessment of Psychological

Symptoms-34. $S E=$ Standard error. ${ }^{* * *} p<0.001$

${ }^{a}$ Results listed in table were generated using robust standard errors. 
the model. The Unconditional Growth Model comprised of continuous predictors, Sessions and Session ${ }^{2}$, were entered at Level 1 (session level) to obtain an average baseline post-test ORS score and average linear and quadratic growth rate for the sample. For this first model, Level 2 (group level) was empty. This model revealed that the average post-test ORS score was 26.31, and clients in the overall sample demonstrated a significant positive linear growth rate $\left(\beta_{10}=0.84, S E=0.09, p<.05\right)$, indicating that an average growth of 0.84 ORS points was observed for each session attended. Clients in the overall sample also demonstrated a significant positive non-linear growth rate $\left(\beta_{10}=0.32\right.$, $S E=0.16, p<.05)$, indicating that clients achieved a quadratic growth rate of an average of 0.32 ORS points for each session attended.

A Conditional Growth Model (i.e., model with treatment conditions added) was constructed by first adding two predictors at Level 2 (group level), to determine if Feedback improved the rate of growth in comparison to clients in the TAU condition. All sessions were centered at Session 1. The dichotomous predictor, Feedback, was added to estimate linear change. Feedback*Session was added to determine quadratic change by treatment condition. An analysis of this Conditional Growth Model revealed that Feedback clients experienced linear growth at a rate of 1.04 ORS points, quadratic growth of -0.14 ORS points, none of which reached statistically significant differences $(p>0.05)$. Therefore, only the linear model was used to evaluate hypothesis two (see Table 3.5). Results indicate that clients in the Feedback condition did not experience improvement more quickly than clients in the TAU condition.

A Chi-square statistic was used to evaluate the deviance statistics from the Unconditional Growth Model (2249.31 with two parameters) to the Conditional Growth Model (2244.01 with two parameters), which concluded that the addition of the Feedback 
variable when controlling for the initial treatment functioning score did not contribute to the overall fit of the model $\left(X^{2}[0, N=85]=5.30, p>.05\right.$; see Table 3.5 for additional details).

Overall, results suggest that clients in this sample evidenced a statistically significant linear and non-linear growth rate across the duration of group therapy. However, Hypothesis 2 was not supported given that clients in a Feedback condition did not achieve a rate of growth that was statistically significant different from those in the TAU condition. More research may be needed to understand what factors accounts for the $50 \%$ of group level variance if not the treatment conditions.

Hypothesis three. I hypothesized that clients in a Feedback condition would achieve higher rates of reliable and clinically significant change. Chi-square analyses revealed statistically significant differences, $\chi^{2}(1, N=85)=7.8, p=.043$ (two-tailed), $95 \%$ CI $[0.38,0.48]$ or $p=0.005$ (one-tailed), 95\% CI [0.004, 0.007], between clients in the Feedback and TAU conditions on classifications based on changes in pre-post ORS scores. Specifically, significant differences were found for clinically significant change (e.g., client improvements of at least 5 points that crosses the clinical cutoff of 25 points on the ORS) and clinically significant change (see Table 3.6).

As can be seen in Table 3.6, the majority of clients in both treatment conditions evidenced little reliable or clinically significant change on the CCAPS-34 Distress Index. A Chi-square analyses, $\chi^{2}(2, N=76)=0.53, p=.77$ (two-tailed) found no statistically differences for reliable or clinically significant change across treatment conditions. Results of Chi-square analyses partially supported hypothesis 3. Clients in the Feedback condition were noted to have achieved statistically significantly higher percentages of 
Table 3.5

Fixed and Random Effect Estimates for Two-level Multilevel Growth Models for

$\operatorname{ORS}(N=85)$

\begin{tabular}{|c|c|c|c|}
\hline Parameter & $\begin{array}{l}\text { Base model } \\
\text { (Unconditional } \\
\text { growth } \\
\text { model 1) }\end{array}$ & $\begin{array}{c}\text { Model one } \\
(\text { Unconditional } \\
\text { growth model 2) }^{a}\end{array}$ & 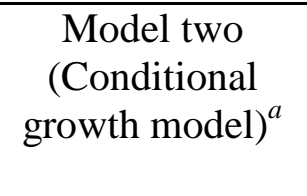 \\
\hline Fixed effects & Coefficient $(S E)$ & Coefficient (SE) & Coefficient $(S E)$ \\
\hline $\begin{array}{l}\text { Intercept: Mean } \\
\text { ORS }\left(\beta_{00}\right)\end{array}$ & $26.05(0.91)^{* * *}$ & $26.31(0.90)^{* * *}$ & $26.31(0.90) * * *$ \\
\hline Session $\left(\beta_{10}\right)$ & & $0.84(0.12)^{* * *}$ & $0.84(0.12) * * *$ \\
\hline $\operatorname{Session}^{2}\left(\beta_{11}\right)$ & & $0.32(0.16)^{*}$ & $0.32(0.16)^{*}$ \\
\hline Feedback $\left(\beta_{01}\right)$ & & & $1.14(1.78)$ \\
\hline Random effects & Coefficient $(S D)$ & Coefficient $(S D)$ & Coefficient $(S D)$ \\
\hline $\begin{array}{l}\text { Session intercept } \\
\text { variance }\left(\sigma^{2} \varepsilon_{\text {session }}\right)\end{array}$ & $33.17(5.76)$ & $33.78(5.81)$ & $34.17(5.85)$ \\
\hline $\begin{array}{l}\text { Group intercept } \\
\text { variance }\left(\tau_{\text {group }}\right)\end{array}$ & $32.97(5.74)$ & $26.47(5.14)$ & $26.49(5.14)$ \\
\hline \multicolumn{4}{|c|}{ Note. $O R S=$ Outcome Rating Scale. $S E=$ Standard error. $S D=$ Standard } \\
\hline
\end{tabular}


reliable and clinically significant change as compared to the TAU condition on the ORS. However, these results were not replicated with the CCAPS-34 Distress Index.

Hypothesis four. I hypothesized that clients in the Feedback condition would attend more sessions overall, drop out and deteriorate at a lower rate, and be prevented from premature termination (dropping out before reaching the clinical cutoff of 25 on the ORS) compared to clients in the TAU condition. On average, clients attended 7.33 sessions $(S D=2.78$; range $=2-11)$. Clients in the Feedback condition attended statistically significantly more sessions than clients in the TAU condition (Feedback: 8.05 versus 6.56 in TAU, $t(83)=2.54, p<.05$, two tailed, 95\% CI [0.32, 2.65]. As can be seen in Tables 3.6 and 3.7, approximately the same number of clients deteriorated between conditions, meaning their final scores on the ORS and CCAPS-34 Distress Index were significantly lower, that is, exceeded respective cut scores derived from the RCI on each measure than when they started group treatment. However, results suggested that the rate by which clients dropped out of group therapy (i.e., did not attend at least $75 \%$ of group treatment sessions) was significantly different across treatment conditions. These results yielded an overall dropout rate of $34 \%$ in the Feedback condition and $56 \%$ in the TAU condition $\left(\chi^{2}[1, N=85]=4.16, p=.04\right)$. Follow-up analyses revealed that clients who dropped out of group therapy did not significantly differ from completers on initial level of functioning on ORS or CCAPS-34 Distress Index scores or on demographic variables (i.e., age, race/ethnicity, gender identity, sexual identity, relationship status). Finally, a relatively similar number of clients terminated prematurely (i.e., dropping out of therapy before reaching the clinical cutoff score of 25) in both conditions (6 clients in the Feedback condition and 7 clients in the TAU condition). 
Table 3.6

Percentage of Clients who Achieved Clinically Significant Change in Feedback and

TAU Conditions on the ORS $(N=85)$

\begin{tabular}{lcccc} 
& $\begin{array}{c}\text { Feedback condition } \\
(n=44)\end{array}$ & $\begin{array}{c}\text { TAU condition } \\
(n=41)\end{array}$ \\
& $n$ & $\%$ & $n$ & $\%$ \\
\multicolumn{1}{c}{ Classification } & 3 & 6.82 & 2 & 4.88 \\
\hline Deteriorated & 9 & 20.45 & 20 & 48.78 \\
No change & 14 & 31.82 & 7 & 17.07 \\
Reliable change & 18 & 40.90 & 12 & 29.27 \\
Clinically significant change & & & &
\end{tabular}

Note. ORS = Outcome Rating Scale. TAU $=$ Treatment as usual. $\chi^{2}(1, N=85)=$

$7.8, p=.043$ (two tailed). 
Table 3.7

Percentage of Clients who Achieved Clinically Significant Change in

Feedback and TAU conditions on the CCAPS-34 Distress Index $(N=76)$

$$
\begin{array}{cc}
\text { Feedback condition } & \text { TAU condition } \\
(n=40) & (n=36)
\end{array}
$$

\begin{tabular}{lcccc}
\hline Classification & $n$ & $\%$ & $n$ & $\%$ \\
\hline Reliably improved & 6 & 13.64 & 6 & 14.63 \\
Reliably deteriorated & 1 & 2.70 & 2 & 4.88 \\
No change & 28 & 63.64 & 33 & 80.49
\end{tabular}

Note. TAU = Treatment as Usual Condition. CCAP-34 = Counseling Center

Assessment of Psychological Symptoms-34. ${ }^{*} \chi^{2}(2, N=76)=.534, p=.766$

(two-tailed). 
An exploratory analysis examining whether more clients considered "at-risk" for premature termination were retained in treatment across conditions was also conducted. After session 3, 10 clients in the TAU condition and 13 clients in the Feedback condition were considered to be at-risk of terminating prematurely; however, these clients remained in treatment throughout its intended duration. After session 6, approximately 10 clients in the TAU condition and 6 clients in the Feedback condition were considered to be atrisk. This result seems noteworthy in that approximately the same number of clients in the TAU condition would have been signaled as being at-risk at both sessions 3 and 6; however, after clinicians in the Feedback condition were provided a signal at session 3 , the number of clients who were at risk at session 6 were nearly reduced by half.

Results from analyses mostly supported hypothesis 4 , in that more clients in the Feedback condition attended significantly more sessions and fewer dropped out of group therapy at a significantly lower rate compared to clients in the TAU condition. Hypothesis four was not supported in that deterioration rates did not differ between treatment conditions. Approximately the same number of clients prematurely terminated from group therapy across condition; however, fewer clients in the Feedback condition were considered at-risk of premature termination at session 6.

\section{Summary}

Results from statistical analyses provide support for the overall efficacy of client feedback in group psychotherapy as well as retention. A medium effect size was also noted regarding the magnitude of change for client feedback. In addition, clients in a Feedback condition experienced significantly improved treatment gains as well as higher rates of reliable and clinically significant change. Unfortunately, clients in a Feedback condition did not improve in fewer sessions compared to TAU. Effects on retention were 
also noteworthy. Clients in a Feedback condition attended statistically significantly more sessions and dropped out at a lower rate. Although approximately the same number of clients in each treatment condition deteriorated and prematurely terminated from treatment, fewer clients in the Feedback condition were considered at risk of premature termination after session 3. Several similarities and differences yielded between results of this study and previous research are described in more detail below. 


\section{Chapter Four: Discussion and}

\section{Conclusions}

In this study, I evaluated the efficacy of client feedback in group psychotherapy in a naturalistic setting. Findings from previous studies have suggested that client feedback reduces deterioration, dropout, and premature termination rates and improves client outcome in both individual (Miller et al., 2006; Reese et al., 2009) and couples (Anker et al., 2009; Reese et al., 2010) therapy formats; however, whether the benefits of client feedback using PCOMS extend to a group therapy format is unknown. Only one published study to this point (Davies et al., 2008) has evaluated a client feedback intervention in a group format and the results were not positive. However, this study differs in that client outcome and the group alliance was monitored each session and a signal alarm system was provided to clinicians to alert them to clients who were at risk of premature termination. The current study yielded four main findings that are discussed below.

\section{Efficacy of Client Feedback}

Positive evidence regarding the efficacy of client feedback in group psychotherapy was a primary finding of this study with regards to both degree of difference and magnitude of change. Clients in the Feedback condition experienced statistically significant pre-post treatment gains on the ORS compared to group clients in the TAU condition when controlling for their initial functioning. Positive results found with the ORS in this study are inconsistent with the only other published study that evaluated the efficacy of client feedback in group psychotherapy using a measure of the group climate (Davies et al., 2008). Not only did Feedback contribute to statistically significant pre-post gains compared to TAU, the magnitude of change was also 
noteworthy. Calculations revealed that within group ES's for both treatment conditions were large (Feedback: $d=0.90$; TAU: $d=0.74$ ). A medium between treatment condition ES of $d=0.35$, and an effect of feedback, was yielded when initial treatment functioning was controlled for on the ORS, which was comparable to other medium ES's noted in previous PCOMS studies with couples $(d=0.50$, Anker et al., 2009; $d=0.48$, Reese et al., 2010). Interestingly, when initial treatment functioning was not controlled, a between group ES of $d=0.44$ was yielded, which is identical to findings noted in previous client feedback research conducted by Lambert (2001a; $d=0.44$ for clients in a Feedback condition who were NOT). To provide further context, the ES for feedback was smaller than in other studies evaluating the general efficacy of group psychotherapy $(d=0.58$; Burlingame et al., 2003); however, this ES was compared to a waitlist control, which may have inflated the ES finding in Burlingame et al.'s meta-analysis.

Results differed when both degree of difference and magnitude of change were evaluated using a second outcome measure. Although results were positive for client feedback efficacy on the ORS, there was not a predicted statistically significant pre-post difference between treatment conditions on the CCAPS-34 Distress Index. However, ES's noted with the CCAPS-34 Distress Index were small to medium. There is perhaps one reason for the different results across outcome measures used in this study. The items that comprise the Distress Index on the CCAPS-34 are related to overall distress as connected to specific symptoms measured by other domains (e.g., Depression, Generalized Anxiety) and may not capture the full range of distress experienced by group clients that perhaps are afforded by the broader domains on the ORS. 


\section{Growth Rate}

A second main finding was that clients in the Feedback condition did not demonstrate more improvement in fewer sessions on the ORS than clients in the TAU condition. This result is not consistent with a previous PCOMS study on the effect of feedback on client's rate of change in individual (Reese et al., 2009) and couple therapy (Reese et al., 2010), in which individuals and couples in a Feedback condition experienced statistically significantly more change in fewer sessions than those in the TAU condition. However, this hypothesis has less empirical support. Rate of change is likely influenced by multiple factors. The composition and rationale for the group needs to be considered with regards to expectations for immediate change to global psychological functioning. To address problems that have a significant or longstanding interpersonal dimension may carry a different expectation in that rapid change may not be reasonable for some in the amount of time provided. Given that interpersonal concerns can be more chronic in nature, client related distress perhaps may take longer to resolve than the "doses" provided in this study. In addition, a majority of clients in this study already received individual therapy prior to attending group therapy and their initial high distress levels may have been reduced depending on the recency of their experience.

\section{Clinically Significant Change}

A third finding based on results from hypothesis 3 was that more clients in the Feedback condition achieved significantly more reliable change (31.82\% vs. $17.07 \%)$ and clinically significant change (40.9\% vs. $29.27 \%$ ) compared to clients in the TAU condition on the ORS. The percentage of clinically significant change is almost identical to results yielded in previous studies evaluating the efficacy of Feedback using PCOMS with couples (40.8\%; Reese et al., 2010). In addition, twice as many clients in the 
Feedback condition achieved reliable improvement than those in the TAU group $(n=14$ versus $n=7$ ), which is similar to findings by Reese et al. (2009). A larger percentage of clients in the TAU condition also remained unchanged according to their outcome scores on the ORS compared to the Feedback condition, meaning that fewer clients in the TAU condition achieved change from pre to post that exceeded 5 ORS points. This finding is also similar to results found in the study conducted by Reese et al. (2010), in which more clients in the TAU condition remained unchanged according to their outcome scores on the ORS.

\section{Retention}

The fourth primary finding based on results from hypothesis 4 was that client feedback appears to have a positive effect on retention. Clients in the feedback condition were found to attend statistically significantly more sessions $(M=8.05 ; S D=2.95)$ than clients in the TAU condition $(M=6.56 ; S D=2.29)$. This is similar to results from Slade and colleagues (2008) that suggested clients in their Feedback conditions on average attended 1.5 more sessions than those in the TAU condition. Results regarding retention in this study are significant given the continuous assessment literature suggests that clients who attend more treatment sessions tend to experience more improvement in psychotherapy (Howard et al., 1986). Drop-out rates were also significantly different for clients in the Feedback condition (34\%; versus those in the TAU condition at $56 \%$ ), which is at the lower end of the average drop-out rate reported in the general psychotherapy outcome literature (Lambert \& Ogles, 2004; Wierzbicki \& Pekarick, 1993). Not consistent with previous research is that approximately the same number of clients prematurely terminated from therapy across treatment. This result differs from that of Lambert et al. (2001a, 2002) in that fewer clients prematurely terminated in a 
Feedback condition. However, an exploratory analysis revealed that nearly $50 \%$ fewer

clients who were considered at-risk of premature termination from treatment at session 3 in the Feedback condition were considered at-risk at session 6, suggesting that clients were no longer at risk of dropping out of treatment once clinicians were alerted to them. Results from this study suggest that using client feedback may be one intervention that contributes to improving retention rates in group psychotherapy as well as reducing the likelihood that clients are at-risk for treatment failure.

\section{Study Limitations}

Limitations noted in this study span across components of the naturalistic setting used to implement the study, the intervention chosen, as well as the data collected/measurement issues.

Naturalistic setting. Three limitations should be noted related to study design given the naturalistic setting. First, randomization at the client level, or individually, to group treatment conditions was not feasible. Both students and co-leaders' schedules made completely randomizing them to groups difficult if not impossible. Despite this limitation, randomization was successful, according to the lack of differences noted between groups on initial functioning. Second, groups at the counseling center do not have a structured pre-group selection measure to verify the likelihood for client improvement in group therapy as is recommended by the group practice evidence-based guidelines (Strauss, Burlingame, \& Bormann, 2008). Given that clients were approved for inclusion based on a wide variety of criteria and based on clinicians' subjective judgment, client change may be related to their level of appropriateness for group therapy. Last, the training provided to co-leaders at this Center encouraged them to open group with a check in (e.g., clients update the group on how they doing that day) and check out 
(e.g., ask them how they are feeling as they leave group that session) procedures and was not modifiable by research personnel given the training component involved with doctoral students. Although there was no way that co-leaders could have monitored members' answers or treatment progress formally from week-to-week based on this process, it is possible that the attempts by co-leaders to assess their group members' progress may have confounded the TAU condition. The study design may have been enhanced if clinicians in the TAU condition did not generate any discussion about client progress in the group, even on an informal basis.

Intervention. A primary aspect that may have influenced study findings was related to the strength of the PCOMS intervention, or independent variable, delivered in the group format. In previous PCOMS efficacy studies, the feedback intervention is likely stronger given that the therapist is able to spend more time addressing the client feedback for the individual or couple. The group format is more complex in that coleaders are tracking not only individualized feedback on what is needed to help them improve in therapy but also monitoring the group as a whole. Pragmatically, simply less time is available to discuss and process the client feedback measures in a group psychotherapy format. Second, although clinicians in the Feedback condition received client progress updates in a number of ways (e.g., verbally and graphically, via signal to alert them to clients who were at-risk of deteriorating or dropping out) and clients received an opportunity to discuss their progress and view it graphically each week, pragmatically less time was available to address the needs of each group member. The level of depth and time it takes to process client feedback in a group may have differed from that which both individual and couple therapy affords. An additional limitation is that although research personnel closely monitored the delivery of client feedback 
measures, CCAPS-34 administrations, and the presence of co-leaders discussions on how they were using the client feedback and information from the signal system in group supervision, treatment fidelity of the degree to which co-leaders implemented group member's feedback was not formally monitored or tracked in this study outside of verbal agreements made by group co-leaders who affirmed they were adhering to the protocol in group supervision. The study design may have been enhanced by more formally monitoring fidelity to treatment through observation on video recordings or developing a rating form for clinicians to assess their adherence to the feedback protocol.

Data. There are six measurement or data limitations of note. First and foremost, the sample of this study was smaller than expected given that more groups $(n=2)$ were scheduled during summer months but were never generated due to a lack of client interest. An a priori power analysis conducted indicated approximately 120 clients were needed for a level of power $(\beta=.80)$ appropriate for this study to detect an ES of $d=0.50$. The post hoc power analysis concluded power $(\beta=.69)$ was insufficient to detect a medium ES $(d=0.50)$. The sample size may have unduly influenced the results of this study and the results should, therefore, be interpreted with caution. Second, although the purpose of the study was for clients to provide feedback to their co-leaders via the PCOMS assessments provided, these measures were still self-report measures of client functioning. Self-report assessment measures are generally known to inherently have some degree of bias towards social desirability and lack validity in how that client may be objectively assessed (Barker, Pistrang \& Elliott, 2005). Third, although the number and adequacy of measures were appropriate for this study, results may have been enhanced by additional measures regarding interpersonal relationship functioning or a different measure of the group alliance that may incorporate additional aspects of group level relationships such as 
the Group Questionnaire (GQ; Burlingame, McClendon, \& Alonso, 2011), which was unavailable at the beginning of this study. It is possible that the outcome measures did not fully capture the specificity of client change in group outside of general psychological distress. Adding additional or lengthier measures in this study would not have been feasible given the busy naturalistic setting; however, the study results may have been enhanced by such measures. Fourth, as is typical with naturalistic studies, missing data were observed between pre and post-test observations. Not monitored in the study were reasons why clients missed sessions or reasons for their premature termination, though common self-reports they shared with group leaders were, "I had [specific task] that I need to do and will be unable to attend group today," family emergencies, called in to work unexpectedly, "I forgot," or "I don't think this [group] is the place for me." More formally understanding client dropout or missed sessions may enhance understanding of why clients leave group treatment. A sample question to ask in future studies is, do clients leave on average because they feel improved or are their needs not being met in treatment? Fifth, some group members who were absent between the pre and post-test on a week when the CCAPS-34 was provided were not always administered the assessment the following week. Ensuring that clients who missed their CCAPS-34 week and provided with a follow up administration ended up being more cumbersome and not monitored well in this study. Although missing data are generally problematic, hierarchical linear modeling (HLM) data analytic methods are designed to handle missing data using an estimation method that does not bias results (Raudenbush \& Bryk, 2002). For example, HLM software utilizes restricted maximum likelihood estimation techniques for parameter estimates, meaning that missing parameter estimates are generated based on the likely value given observed scores (McKnight, McKnight, Sidani, 
\& Figueredo, 2007). Last, two groups had at least one session in which the measures were not administered according to protocol. One was a TAU group that reported "forgetting" to administer the ORS at session 3 and one Feedback group was unable to get the CCAPS-34 administered for the final session due to a miscommunication with the front desk staff at the Center.

A final limitation is related to generalizability. At least two primary factors regarding the data are considered to be threats to external validity: the composition of sample and the university setting. The sample in this study lacked racial and ethnic diversity, though the percentages of demographic information of the student population in this sample were similar to the student population characteristic of the university. Second, being on a university campus also suggests that the participants in this study were well educated individuals whose results may not generalize well to a community mental health agency or other treatment settings.

\section{Implications and Future Recommendations}

Results yielded from this study inform both the implications and future recommendations.

Implications. Results of this study may provide implications for both the research and practice of implementing client feedback in group psychotherapy. Two implications from this study are noted here. First, the protocol and logistics for this study may have implications for implementing a client feedback system with groups in naturalistic settings. As can be seen from the procedures section, multiple layers exist when developing a protocol that includes repeated measures, experimental conditions, and a clinical sample. For example, administrative permission was needed from the director of the agency, multiple meetings were held to coalesce feasible research and clinical 
procedures, and consistent oversight was required on a weekly basis to monitor clients' treatment needs while also attending to a research protocol that required co-leaders to complete multiple tasks each session. Procedures included the protocol outlined above may provide some foundational ideas as to how an agency may be able to incorporate client feedback in a group psychotherapy program. Second, this study extends the client feedback literature to include results on the efficacy of feedback in group psychotherapy. Group clients in a Feedback condition were predicted to have statistically significantly higher pre-post gain scores in treatment as well as evidenced higher retention rates, indicating that the practice of monitoring client outcome and the group alliance in group therapy enhances group treatment compared to TAU. Given that group therapy is a widely utilized treatment modality in community resources due to its cost and personnel efficiency (Taylor et al., 2001), utilizing client feedback may further enhance client outcomes in this modality as well as retain clients through the intended duration of group treatment.

Future recommendations. I have nine recommendations for future studies. First, I recommended that additional outcome measures that comprise relationship functioning be included in the data collection process given that interpersonal process groups are focused in this way and clients are referred to these groups for relationship concerns. Although the CCAPS-34 has a subscale on Social Anxiety, it was not incorporated into the current analysis but may be evaluated in future studies. I hope that in future work, the Group Questionnaire (Burlingame et al., 2011) could be administered to broaden the conceptualization and assessment of the therapeutic relationships found in group beyond that of the therapeutic alliance as it is known in the individual therapy literature. Second, future research may also be developed around how monitoring the 
group alliance influenced client outcome and at what point in the group does the group alliance (either positive or negative) predict or affect client outcome. Third, I also recommend that additional data be collected to further evaluate findings with a larger and more diverse sample. A larger sample will allow for a higher degree of power to reject the null hypothesis (reduce Type II error), as was problematic in this study. A more diverse sample of clients from settings outside of a university counseling center may also improve generalizability of findings from this study but also, in general, for client feedback research.

Fourth and fifth recommendations are related to formalizing a check of treatment integrity to estimate: (a) the level of fidelity to the intervention, as well as (b) to better understand how the process of client feedback is implemented. More formal methods of monitoring the implementation of the intervention may be warranted. For example, observing the video recordings of group sessions may provide an understanding of the strength of the intervention implemented in groups as well as how the intervention could be delivered more effectively. For example, is more time needed for clients to process their progress for client feedback to be more effective in group? Studying clients' and coleaders' perceptions of feedback in the group may elicit an understanding or further questions to highlight the processes underlying client feedback that have contributed to improved client outcome in individual (Lambert et al., 2001a, 2002; Miller et al., 2006; Reese et al., 2009) and couples psychotherapy (Anker et al., 2009; Reese et al., 2010). Understanding these processes may also provide insight into how clients perceive providing feedback, how they used the measures, and what sharing their results in a group format was like for them to better tailor the intervention to this treatment modality. 
Sixth and seventh recommendations for future research are related to exploring how aspects of the study protocol that were out of the research personnel's control influenced the current study findings. For example, it may beneficial to more fully evaluate whether the check-in/check-out procedures conducted in the TAU condition makes approximately as much difference in client outcome as more formally monitoring client outcome. Evaluating this may be possible by isolating groups to a client feedback condition, check in/check out procedure condition, and a group in which no question regarding their perceptions of their progress or relationships in the group may provide insight on what degree of client monitoring provides enhanced client outcome in group therapy. In addition, I hope that clients might have more time to expand upon their progress on the outcome and group alliance to more fully explore how the group can help support those needs during the session. Given that a short duration of time was allotted for this intervention by the center in this study, I am curious to evaluate if client feedback would be stronger in the group format if more time was given to the process of clients further expanding on their needs related to their outcome and the group alliance each week.

Exploring the effectiveness and feasibility of PCOMS in groups using the computer-based version of the PCOMS program versus the paper-pencil version may also warrant study and is an eighth recommendation. I hoped to obtain research funding to purchase and utilize the computer-version of PCOMS so that clinicians could receive the signal system more immediately in the session. Unfortunately purchasing the program was not possible. However, future research may benefit from evaluating whether the computer version improves effectiveness given the signal would be even more immediate. Utilizing the PCOMS computer version may also warrant study to evaluate whether 
feasibility is enhanced, given that the paper-version may be more cumbersome for clients to respond to, score, and plot at the beginning of group.

My ninth recommendation is for studies to evaluate whether to whom feedback is delivered is important. We know that the individual psychotherapy outcome literature evidences mixed findings (Harmon et al., 2005; Hawkins et al., 2004). For example, would clients' outcomes have been similar if only the co-leaders received their feedback

and signal system? It is also of interest to evaluate the signal system that alerts co-leaders to client progress to help to understand how to strengthen the intervention at the group level as well as who benefits from the signal in a group format.

\section{Conclusions}

Overall, much is to be learned about extending the idea of monitoring outcome and alliance to groups, which is recommended as an evidence-based practice (APA Presidential Task Force on Evidence Based Practice, 2006) from both pragmatic and research perspectives. Although preliminary evidence found in this study supporting client feedback shows promise for utilizing this practice in group therapy, additional research is warranted with a larger more diverse sample and enhanced study design to clarify findings. These preliminary results suggest that client feedback yields a noteworthy and improves retention in group psychotherapy, extending findings from both individual (Miller et al., 2006; Reese et al., 2009; Slade et al., 2008) and couple (Anker et al., 2009; Reese et al., 2010) PCOMS efficacy studies. I hope additional research contributes evidence that client feedback enhances client outcome and attendance in group psychotherapy. 


\section{Appendix A:}

\section{Group Leader Demographic Form}

Gender: Man:

Woman:

Self-identify (Write in):

Age:

Ethnicity:

Caucasian

African American

Hispanic/Latino

Asian/Pacific Islander
Multiracial
Self-identify:

Highest degree earned (please specify degree program):

Number of years providing group

therapy:

Please provide an approximate number of interpersonal process groups you have led/colead:

Please check the line below that best describes your theoretical orientation: Cognitive/Behavioral

Psychodynamic

Psychoanalytic

Existential/Humanistic

Interpersonal Process

Narrative

Integrative (please describe predominate theories): 


\section{Appendix B:}

1. Date of Birth

\section{Client Demographic Form}

2. Gender Identity

3. Race/Ethnicity
a. International student?
b. Country of Origin

4. Sexual Orientation

5. Relationship status

6. Academic Status

7. What is your Major?
a. GPA
b. Semester Credits

8. Housing

a. Living with? (Alone, spouse, partner, significant other, roommate(s), children, parents or guardian, family/other

b. Living with Other: (write in)

9. Extra-curricular hours:

10. Registered with disability services? (Y/N)

a. If yes, specific for what disorder

11. Athlete?

12. First Generation?

13. Military Service?

14. Financial stress now? (rarely stressful, sometimes stressful, often stressful, Always, stressful) 
15. How did you learn about the Counseling Center?

16. Binge drinking frequency?

17. Smoked Marijuana (Frequency)

a. Please check any other drugs you have used (Cocaine/Crack, Ecstasy, LSD, PCP, Heroin, Methamphetamine, Inhalants, Prescription drugs (nonmedical use), and Other)

18. Please indicate if/when you have had the following experiences:

a. Prior Counseling (None, Before College, After College, Both)

b. Prior meds ((None, Before College, After College, Both)

c. Prior hospitalization (How many)

d. Need to reduce drugs and alcohol (How many, Last time)

e. Others concern alcohol (How many, Last time)

f. Prior drug and alcohol treatment? (How many, Last time)

g. Self-injury (How many, Last time)

h. Considered suicide (How many, Last time)

i. Harmed another (How many, Last time)

j. Unwanted Sexual Experience (How many, Last time)

k. Harassment/Abuse (How many, Last time)

1. PTSD Experience (How many, Last time, Description)

19. Current Medications

20. Briefly describe what brings you to the counseling center.

21. Health problems? (Yes/No)

a. If yes, please describe. 


\section{Appendix C:}

\section{Computer Script}

The Counseling Center compiles data on client demographics, issues of concern and service outcome. This data is used for planning services, institutional evaluation and also for published research including participation in a national research project at Penn State University designed to improve our services and expand the knowledge about college student mental health. Data is stripped of all personally identifying information and then combined to create an anonymous pool so no client's confidential information can be specifically identified. Because data cannot be linked to specific individuals, there are virtually no risks in contributing data. With your permission we would like to contribute your data both to the national research project and for research use at UKCC. Your decision is voluntary. You have the right to request that your data be excluded from published research, and your decision will not affect the services you receive. If you have questions or concerns, you may contact Dr. Nathaniel Hopkins or the UKCC Director, Dr. Mary Bolin.

Will you allow your anonymous responses to be contributed? Yes/No 


\section{Appendix D:}

\section{Screening Script (Feedback condition)}

We are conducting a research study to evaluate our group therapy program. Your responses on two brief surveys each week will help us understand how you are feeling personally and as a member of the group every session. Your information will be used to help us better understand how we can help you work towards your goals and have the best possible experience in group therapy. We will ask you to respond to a short survey called the Outcome Rating Scale (ORS) at beginning of each session that will ask you about how you are doing in three domain areas of your life: Personally, Interpersonally, Socially, and Overall (can show client how to respond to the measure at this point). You will then use a scoring overlay (transparency) to score your own ORS and plot your score on a graph to see how you are progressing over the course of therapy. If you are not progressing as you hoped, we would like to help you. To do so, your group leaders will check in with you about your progress at the beginning of each group session. You

will also be asked to respond to the Group Session Rating Scale (GSRS), a brief measure of how you are feeling as a group member. You will be asked to respond to, score, and graph your Total Score at the end of each session so you can see your progress. Lastly, as is procedure for UKCC, you will be asked to respond to the CCAPS every three weeks when you check in to get a more in depth understanding of how you are doing as you progress in therapy. It is really important that you check in at the front desk before you arrive in group. If you are running late, you can simply fill out any surveys you missed at the end of group. If you have chosen to opt out of research on the informed consent/information form you filled out at intake, you will still be asked to complete the 
surveys each week as it is our routine procedure; however, your information will not be used in any way in the research study. Do you have any questions? 


\section{Appendix E:}

\section{Screening Script \\ (Control condition)}

We are conducting a research study to evaluate our group therapy program. Your responses on a brief survey called the Outcome Rating Scale (ORS) will help us understand how you are feeling each week, and it will help us determine whether group therapy was helpful to you. We will ask you to respond to a short survey at beginning of each session that will ask you about how you are feeling that day on in three domain areas: Personally, Interpersonally, Socially, and Overall. (can explain to them how to respond to the ORS here). We will ask you to simply hand it back in to us after you complete it. If you are ever late to group, we will ask you to fill this out at the end of group before you leave that day. As is procedure for UKCC, you will be asked to take a CCAPS every three weeks when you check in. It is really important that you check in at the front desk before you arrive in group. If you are running late, you can simply fill out any surveys you missed at the end of group. If you have chosen to opt out of research on the informed consent/information form you filled out to at intake, you will still be asked to complete the surveys each week as that is our routine procedure, but your information will not be used in any way in the research study. Do you have any questions? 


\section{Appendix F: Outcome}

\section{Rating Scale}

Outcome Rating Scale (ORS)

Student ID\#:

Group:

Date:

Session\#:

Looking back over the last week, including today, help us understand how you have been feeling by rating how well you have been doing in the following areas of your life, where marks to the left represent low levels and marks to the right indicate high levels. If you are filling out this form for another person, please fill out according to how you think he or she is doing.

\section{Individually}

(Personal well-being)

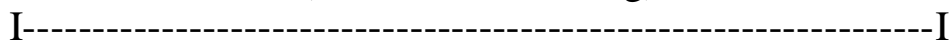

Interpersonally

(Family, close relationships)

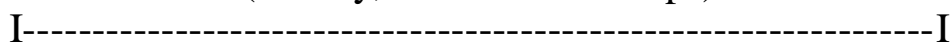

Socially

(Work, school, friendships)

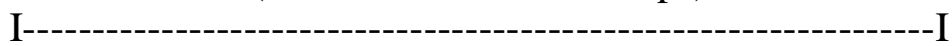

\section{Overall}

(General sense of well-being)

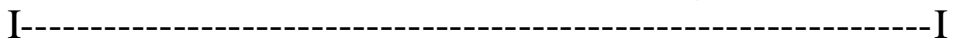

The Heart and Soul of Change Project

www.heartandsoulofchange.com

(C) 2000, Scott D. Miller and Barry L. Duncan 


\section{Appendix G:}

\section{Group Session Rating Scale}

Group Session Rating Scale (GSRS)

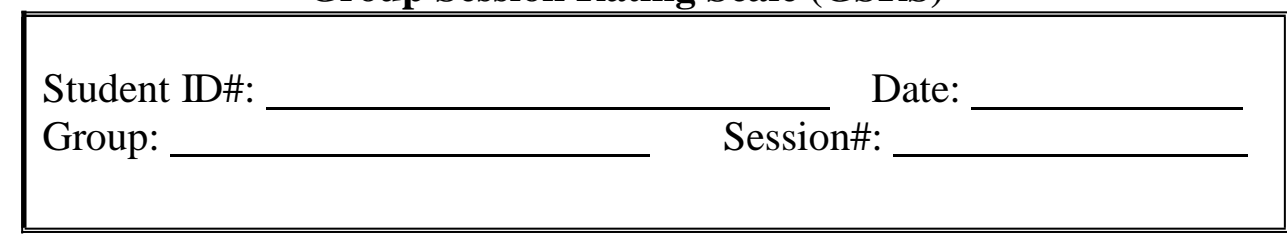

Please rate today's group by placing a mark on the line nearest to the description that best fits your experience.

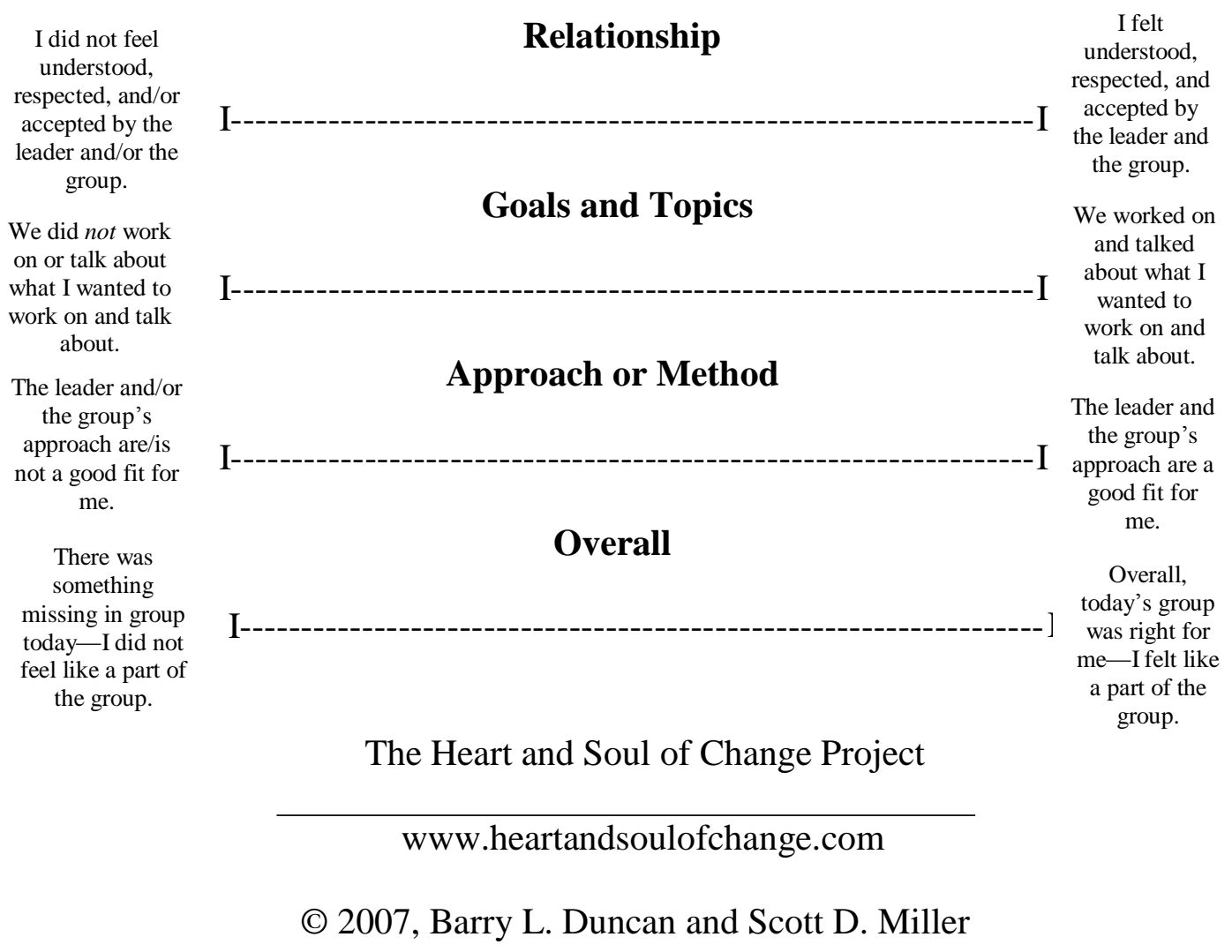




\section{Appendix H:}

\section{Graph for ORS and GSRS}

Client Initials:

OUTCOME RATING SCALE

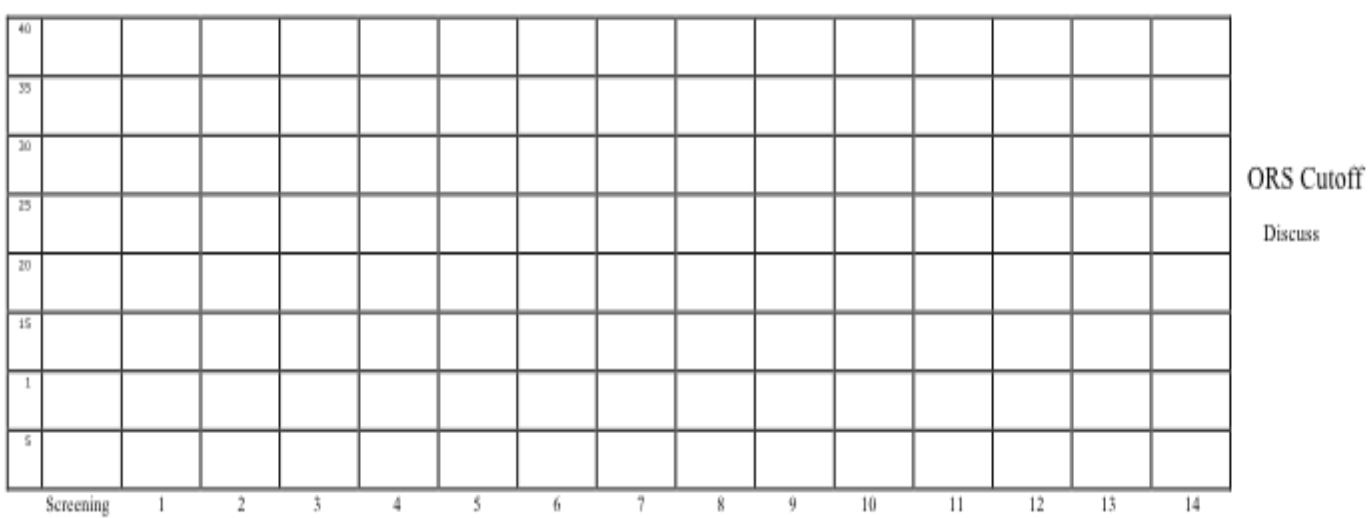

GROUP SESSION RATING SCALE

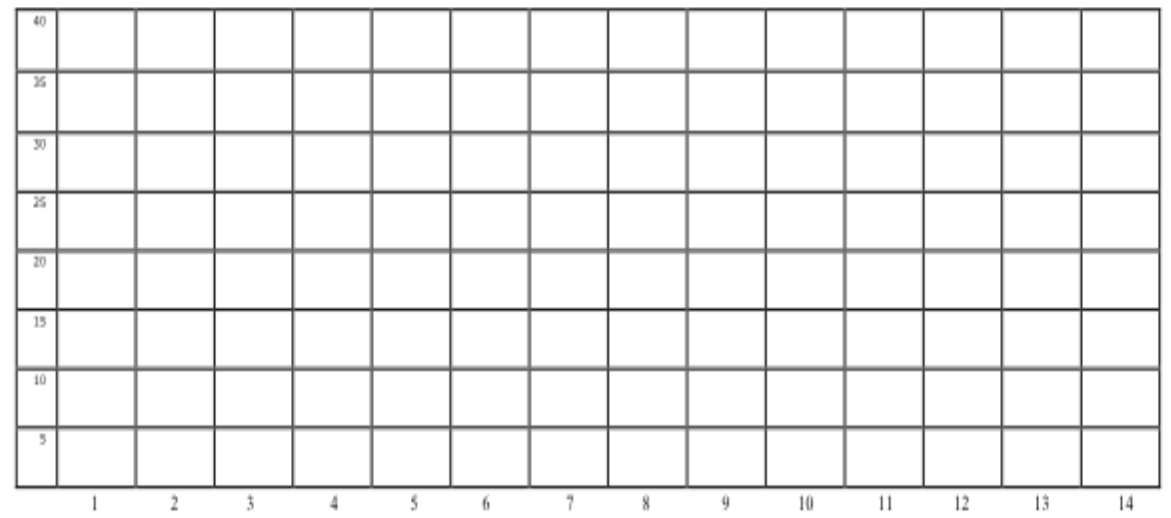

GSRS Cutoff

Discuss 


\section{Appendix I:}

\section{Counseling Center Assessment of Psychological Symptoms - 34}

\section{Counseling Center Assessment of Psychological Symptoms - 34 (CCAPS-34)}

Instructions: The following statements describe thoughts, feelings, and experiences that people may have. Please indicate how well each statement describes you, during the past two weeks, from "not at all like me" (0), to "extremely like me (4), by marking the correct number. Read each statement carefully, select only one answer per statement, and please do not skip any questions.

Student ID Number:

\begin{tabular}{|c|c|c|c|c|c|}
\hline & $\begin{array}{l}\text { Not at } \\
\text { all like } \\
\text { me }\end{array}$ & & & & $\begin{array}{c}\text { Extreme } \\
\text {-ly like } \\
\text { me }\end{array}$ \\
\hline 1. I am shy around others. & 0 & 1 & 2 & 3 & 4 \\
\hline $\begin{array}{l}* 2 \text {. My heart races for no } \\
\text { good reason. }\end{array}$ & 0 & 1 & 2 & 3 & 4 \\
\hline $\begin{array}{l}\text { 3. I feel out of control when I } \\
\text { eat. }\end{array}$ & 0 & 1 & 2 & 3 & 4 \\
\hline $\begin{array}{l}* 4 \text {. I don't enjoy being around } \\
\text { people as much as a I used to. }\end{array}$ & 0 & 1 & 2 & 3 & 4 \\
\hline *5. I feel isolated and alone. & 0 & 1 & 2 & 3 & 4 \\
\hline $\begin{array}{l}\text { 6. I think about food more } \\
\text { than I would like to. }\end{array}$ & 0 & 1 & 2 & 3 & 4 \\
\hline $\begin{array}{l}* 7 . \text { I am anxious that I might } \\
\text { have a panic attack while in } \\
\text { public. }\end{array}$ & 0 & 1 & 2 & 3 & 4 \\
\hline $\begin{array}{l}\text { 8. I feel confident that I can } \\
\text { succeed academically. }\end{array}$ & 0 & 1 & 2 & 3 & 4 \\
\hline *9. I have sleep difficulties. & 0 & 1 & 2 & 3 & 4 \\
\hline$* 10$. My thoughts are racing. & 0 & 1 & 2 & 3 & 4 \\
\hline *11. I feel worthless. & 0 & 1 & 2 & 3 & 4 \\
\hline *12. I feel helpless. & 0 & 1 & 2 & 3 & 4 \\
\hline 13. I eat too much. & 0 & 1 & 2 & 3 & 4 \\
\hline 14. I drink alcohol frequently. & 0 & 1 & 2 & 3 & 4 \\
\hline $\begin{array}{l}* 15 . \text { I have spells of terror or } \\
\text { panic. }\end{array}$ & 0 & 1 & 2 & 3 & 4 \\
\hline $\begin{array}{l}\text { 16. When I drink alcohol I } \\
\text { can't remember what } \\
\text { happened. }\end{array}$ & 0 & 1 & 2 & 3 & 4 \\
\hline *17. I feel tense. & 0 & 1 & 2 & 3 & 4 \\
\hline $\begin{array}{l}\text { 18. I have difficulty } \\
\text { controlling my temper. }\end{array}$ & 0 & 1 & 2 & 3 & 4 \\
\hline
\end{tabular}




\begin{tabular}{|c|c|c|c|c|c|}
\hline 19. I make friends easily. & 0 & 1 & 2 & 3 & 4 \\
\hline $\begin{array}{l}* 20 . \text { I sometimes feel like } \\
\text { breathing or smashing things. }\end{array}$ & 0 & 1 & 2 & 3 & 4 \\
\hline$* 21$. I feel sad all of the time. & 0 & 1 & 2 & 3 & 4 \\
\hline $\begin{array}{l}* 22 . \text { I am concerned that other } \\
\text { people do not like me. }\end{array}$ & 0 & 1 & 2 & 3 & 4 \\
\hline$* 23$. I get angry easily. & 0 & 1 & 2 & 3 & 4 \\
\hline $\begin{array}{l}\text { 24. I feel uncomfortable } \\
\text { around people I do not know. }\end{array}$ & 0 & 1 & 2 & 3 & 4 \\
\hline $\begin{array}{l}* 25 . \text { I have thoughts of ending } \\
\text { my life. }\end{array}$ & 0 & 1 & 2 & 3 & 4 \\
\hline $\begin{array}{l}* 26 . \text { I feel self conscious } \\
\text { around others. }\end{array}$ & 0 & 1 & 2 & 3 & 4 \\
\hline 27. I drink more than I should. & 0 & 1 & 2 & 3 & 4 \\
\hline $\begin{array}{l}28 \text {. I am not ale to concentrate } \\
\text { as well as usual. }\end{array}$ & 0 & 1 & 2 & 3 & 4 \\
\hline $\begin{array}{l}* 29 . \text { I am afraid I may lost } \\
\text { control and act violently. }\end{array}$ & 0 & 1 & 2 & 3 & 4 \\
\hline $\begin{array}{l}* 30 . \text { It's hard to stay motivated } \\
\text { for my classes. }\end{array}$ & 0 & 1 & 2 & 3 & 4 \\
\hline $\begin{array}{l}31 . \text { I have done something I } \\
\text { have regretted because of } \\
\text { drinking. }\end{array}$ & 0 & 1 & 2 & 3 & 4 \\
\hline $\begin{array}{l}\text { 32. I frequently get into } \\
\text { arguments. }\end{array}$ & 0 & 1 & 2 & 3 & 4 \\
\hline $\begin{array}{l}* 33 . \text { I am unable to keep up } \\
\text { my schoolwork. }\end{array}$ & 0 & 1 & 2 & 3 & 4 \\
\hline $\begin{array}{l}\text { 34. I have thoughts of hurting } \\
\text { others. }\end{array}$ & 0 & 1 & 2 & 3 & 4 \\
\hline
\end{tabular}

*denotes items that comprise the Distress Index scale. 


\section{Appendix J:}

\section{Signal Alarm Cards}

No change

(On yellow card)

Clients who have not made a 5-points increase or decreased in the first three sessions are at increased risk of treatment failure. It is recommended that leaders inquire about client's perspectives of therapy and the alliance to determine if changes can be made to the strategy of meeting client's needs and goals.

Reliably deteriorated

(On red card)

Clients who have deteriorated by at least 5-points from their baseline measure are considered reliably deteriorated and are considered to be at risk of dropping out. It is recommended that leaders address the deterioration and inquire about client's perspectives of therapy and the alliance. If clients continue to deteriorate, it is recommended that leaders speak with clients about modifying change strategy to meet client's goals (e.g., augment with medication).

Reliably changed

(On blue card)

Clients who have made at least a 5-point improvement on the ORS have experienced reliable change. It is recommended that leaders reinforce progress and specific changes clients have made and assist clients with further refining their change strategies.

Clinically significant change

(On green card)

Clients who have experienced a 5-point gain and crossed the clinical cutoff of 25 on the ORS are said to experience clinically significant change, meaning clients are experiencing recovery according to scores on this measure. It is recommended that leaders will assist clients in this category refine their changes strategies and focus on preparing them for potential relapses or setbacks. 


\section{References}

Abouguendia, M., Joyce, A. S., Piper, W. E., \& Ogrodnickzuk, J. S. (2004). Alliance as a mediator of expectancy effects in short term group psychotherapy. Group Dynamics: Theory, Research, and Practice, 8, 3-12. doi:10.1037/1089-2699.8.13

American Psychological Association Presidential Task Force on Evidence-Based Practice (2006). Evidence based practice in psychology. American Psychologist, 61, 271285. doi: 10.1037/0003-066X.61.4.271

Anker, M. G., Duncan, B. L., \& Sparks, J. A. (2009). Using client feedback to improve couples therapy outcomes: A randomized clinical trial in a naturalistic setting. Journal of Consulting \& Clinical Psychology, 77, 693-704. doi:10.1037/a0016062

Antonuccio, D. O., Davis, C., Lewinsohn, P. M., \& Breckenridge, J. S. (1987). Therapist variables related to cohesiveness in a group treatment for depression. Small Group Behavior, 18, 557-564. doi: 10.1177/104649648701800409

Aycock, A. C. (unknown). Latent growth curve analysis: A gentle introduction. Unpublished report from Oregon State University. Retrieved from http://oregonstate.edu/dept/hdfs/papers/lgcgeneral.pdf

Baekeland, F., \& Lundwall, L. (1975). Dropping out of treatment: A critical review. Psychological Bulletin, 82, 738-783. doi:10.1037/h0077132

Baker, R. W., \& Siryk, B. (1986). Exploratory intervention with a scale measuring adjustment to college. Journal of Counseling Psychology, 33, 31-38. doi:10.1037/0022-0167.33.1.31

Barker, C., Pistrang, N. \& Elliott, R. (2002). Research methods in clinical psychology ( $2^{\text {nd }}$ ed.). Hobken, NJ: John Wiley \& Sons, Inc.

Beck, A. T., Epstein, N., Brown, G., \& Steer, R. A. (1988). An inventory for measuring 
clinical anxiety: Psychometric properties. Journal of Consulting and Clinical Psychology, 56, 893-897. doi:10.1037/0022-006X.56.6.893

Beck, A. T., Ward, C. H., Mendelson, M., Mock, J., \& Erbaugh, J. (1961). An inventory for measuring depression. Archives of General Psychiatry, 4, 561-571. Retrieved from http://archpsyc.ama-assn.org/

Bordin, E. S. (1979). The generalizability of the psychoanalytic concept of the working alliance. Psychotherapy: Research \& Practice, 16, 252- 260. doi:10.1037/h0085885

Bringhurst, D. L., Watson, C. W., Miller, S. D., \& Duncan, B. L. (2006). The reliability and validity of the Outcome Rating Scale: A replication study of a brief clinical measure. Journal of Brief Therapy, 5, 23-30. Retrieved from http://journalbrieftherapy.com/

Budman, S. H., Soldz, S., Demby, A., \& Feldstein, M. (1989). Cohesion, alliance, and outcome in group psychotherapy. Psychiatry: Interpersonal and Biological Processes, 52, 339-350. Retrieved from http://www.guilford.com/cgibin/cartscript.cgi?page=pr/jnps.htm\&dir=periodicals/per_psych\&cart_id=460889. 26343

Burlingame, G. M., Fuhriman, A., \& Mosier, J. (2003). The differential effectiveness of group psychotherapy: A meta-analytic study. Group Dynamics: Theory, Research, and Practice, 7, 3-12. doi:10.1037/1089-2699.7.1.3

Burlingame, G. M., McClendon, D. T., \& Alonso, J. (2011). Cohesion in group therapy. Psychotherapy, 48, 34-42. doi: 10.1037/a0022063

Chambless D. L., Tran G.Q., \& Glass C. R. (1997). Predictors of response to cognitivebehavioral group therapy for social phobia. Journal of Anxiety Disorders, 11, 
221-240. doi: 10.1016/S0887-6185(97)00008-X

Chapman, C. L., Burlingame, G. M., Gleave, R. M., Rees, F., Beecher, M., \& Porter, G. S. (2012). Clinical prediction in group psychotherapy. Psychotherapy Research, 22, 673-681. doi:1-/1080./10503307.2012.702512

Coe, R. (2002). It's the effect size, stupid: What effect size is and why it is important. Unpublished paper presented at the Annual Conference of the British Educational Research Association, University of Exeter 12-14 September 2002. Retrieved from http://www.leeds.ac.uk/educol/documents/00002182.htm

Cohen, J. (1988). Statistical power analysis for the behavioral sciences $\left(2^{\text {nd }}\right.$ ed.). Hillsdale, NJ: Lawrence Erlbaum Associates.

Crowe, T. P., \& Grenyer, B. F. (2008). Is therapist alliance or who group cohesion more influential in group psychotherapy outcomes? Clinical Psychology and Psychotherapy, 15, 239-246. doi:10.1002/cpp.583

Davies, D. R., Burlingame, G. M., Johnson, J. E., Gleave, R. L., \& Barlow, S. H. (2008). The effects of a feedback intervention on group process and outcome. Group Dynamics: Theory, Research, and Practice, 12, 141-154. doi:10.1037/10892699.12.2.141

Davis, S., Hooke, G. R., \& Page, A. C. (2006). Identifying and targeting predictors of drop out from group cognitive behavioral therapy. Australian Journal of Psychology, 58, 48-56. doi:10.1080/00049530500504096

Dies, R. R., \& Dies, K. R. (1993). The role of evaluation in clinical practice: Overview and group treatment illustration. International Journal of Group Psychotherapy, 43, 77-105. Retrieved from http://www.guilford.com/cgibin/cartscript.cgi?page=pr/jngr.htm\&dir=periodicals/per_psych\&cart_id= 
Duncan, B. L., \& Miller, S. M. (2007). The group session rating scale. Chicago, IL: Authors.

Duncan, B. L., Miller, S. D., Wampold, B. E., \& Hubble, M. A. (2010). The heart and soul of change ( $2^{\text {nd }}$ ed.). Washington D. C.: American Psychological Association. Gaston, L., \& Marmar, C. R. (1994). The California psychotherapy alliance scales. In A. Horvath \& L. S. Greenberg (Eds.), The working alliance: Theory, research, and practice (pp. 85-108). New York, NY: John Wiley \& Sons.

Gaston, L., Marmar, C. R., Gallagher, D., \& Thompson, L. W. (1991). Alliance prediction of outcome beyond in-treatment symptomatic change as psychotherapy processes. Psychotherapy Research, 1, 104-112. doi:10.1080/10503309112331335531

Gillaspy, J. A., Wright, A. R., Stokes, S., \& Adinoff, B. (2002). Group alliance and cohesion as predictors of drug and alcohol abuse treatment outcomes. Psychotherapy Research, 12, 213-229. doi:10.1093/ptr/12.2.213

Greenson, R. R. (1965). The working alliance and the transference neuroses. Psychoanalysis Quarterly, 34, 155-181. Retrieved from http://www.psaq.org/

Hannan, C., Lambert, M. J., Harmon, C., Nielson, S. L., Smart, D. W., Shimokawa, K., \& Sutton, S. W. (2005). A lab test and algorithms for identifying clients at risk for treatment failure. Journal of Clinical Psychology, 61, 155-163. doi:10.1002/jclp.20108

Hansen, N. B., Lambert, M. J., \& Foreman, E. M. (2002). The psychotherapy doseresponse effect and its implications for treatment delivery services. Clinical Psychology: Science and Practice, 9, 329 - 343. doi:10.1093/clipsy/9.3.329

Harmon, S. C., Lambert, M. J., Smart, D. M., Hawkins, E., Nielsen, S. L., Slade, K., \& 
Lutz, W. (2007). Enhancing outcome for potential treatment failures: Therapistclient feedback and clinical support tools. Psychotherapy Research, 17, 379-392. doi:10.1080/10503300600702331

Hatcher, R. L., \& Barends, A. W. (1996). Patients' view of the alliance in psychotherapy: Exploratory factor analysis of three alliance measures. Journal of Consulting and Clinical Psychology, 64, 1326-1336. doi:10.1037/0022-006X.64.6.1326

Hawkins, E. J., Lambert, M. J., Vermeersch, D. A., Slade, K. L., \& Tuttle, K. C. (2004). The therapeutic effects of providing patient progress information to therapists and patients. Psychotherapy Research, 14, 308-327. doi:10.1093/ptr/kph027

Hoffmann, L., Gleave, R., Burlingame, G., \& Jackson, A. P. (2009). Exploring interactions of improvers and deteriorates in the group therapy process: A qualitative analysis. International Journal of Group Psychotherapy. 59,181-199. doi:10.1521/ijgp.2009.59.2.179

Hogue A, Liddle H. A., \& Rowe C. (1996). Treatment adherence process research in family therapy: A rationale and some practical guidelines. Psychotherapy: Theory, Research, Practice, and Training, 33, 332-345. doi: 10.1037/0033-3204.33.2.332

Horne, A. M., \& Rosenthal, R. (1997). Research in group work: How did we get where we are? Journal for Specialists in Group Work, 22, 228-240. doi:10.1080/01933929708415527

Horvath, A. O., \& Greenberg, L. S. (1986). Development and validation of the working alliance inventory. Journal of Counseling Psychology, 36, 223-233. doi:10.1037/0022-0167.36.2.223 
Horvath, A. O., \& Luborsky, L. (1993). The role of the therapeutic alliance in psychotherapy. Journal of Consulting and Clinical Psychology, 61, 561-573. doi:10.1037/0022-006X.61.4.561

Horvath, A. O., \& Symonds, D. B. (1991). Relation between working alliance and outcome in psychotherapy: A meta-analysis. Journal of Counseling Psychology, 38, 139-149. doi:10.1037/0022-0167.38.2.139

Howard, K. I., Kopta, S. M., Krause, M. S., \& Orlinsky, D. E. (1986). The dose-effect relationship in psychotherapy. American Psychologist, 41, 159-164. doi: 10.1037/0003-066X.41.2.159

Howard, K., Moras, K., Brill, P. L., Martinovich, Z., \& Lutz, W. (1996). Evaluation of psychotherapy: Efficacy, effectiveness, and patient progress. American Psychologist, 51, 1059-1064. doi:10.1037/0003-066X.51.10.1059

Hox, J. (2002). Multilevel analysis techniques and applications. Mahwah, NJ: Erlbaum. International Business Machines Corporation (2011). SPSS Statistics for windows (Version 20.0) [computer software]. Armonk, NY: International Business Machines Corporation.

Jacobson, N. S., \& Truax, P. (1991). Clinical significance: A statistical approach to defining meaningful change in psychotherapy research. Journal of Consulting and Clinical Psychology, 59, 12-19. doi:10.1037/0022-006X.59.1.12

Johnson, J. E., Burlingame, G. M., Olsen, J. A., Davies, D. R., \& Gleave, R. L. (2005). Group climate, cohesion, alliance, and empathy in group psychotherapy: Multilevel structural equation models. Journal of Counseling Psychology, 52, 310-321. doi:10.1037/0022-0167.52.3.310 
Joyce, A. S., Piper, W. E., \& Ogrodniczuk, J. S. (2007). Therapeutic alliance and cohesion variables as predictors of outcome in short-term group psychotherapy. International Journal of Group Psychotherapy, 57, 269-296. Retrieved from http://www.guilford.com/cgibin/cartscript.cgi?page=pr/jngr.htm\&dir=periodicals/ per_psych\&cart_id=460889.26343

Lambert, M. (1992). Implications for outcome research for psychotherapy integration. In J. C. Norcross \& M. R. Goldstein (Eds.), Handbook of psychotherapy integration (pp. 94-129). New York, NY: Basic Books.

Lambert, M. J., \& Barley, D. E. (2001b). Research summary on the therapeutic relationship and psychotherapy outcome. Psychotherapy: Theory, Research, Practice, Training, 38, 357-361. doi: 10.1037/0033-3204.38.4.357

Lambert, M. J., Hansen, N. B., \& Finch, A. E. (2001b). Patient-focused research: Using patient outcome data to enhance treatment effects. Journal of Consulting and Clinical Psychology, 69, 159-172. doi:10.1037//0022-006X.69.2.159

Lambert, M. J., Hansen, N. B., Umphress, V., Lunnen, K., Okiishi, J., Burlingame, G. M., \& Reisenger, C. W. (1996). Administration and scoring manual for the $O Q 45.2$. Stevenson, MD: American Professional Credentialing Services.

Lambert, M. J., \& Ogles, B. M. (2004). The efficacy and effectiveness of psychotherapy. In M. J. Lambert (Ed.), Bergin and Garfield's handbook of psychotherapy and behavior change $\left(5^{\text {th }}\right.$ ed.) (pp. 139-193). New York, NY: Wiley.

Lambert, M. J., \& Shimokawa, K. (2011). Collecting client feedback. Psychotherapy, 48, 72-79. doi:10.1037/a0022238

Lambert, M. J., Whipple, J. L., Hawkins, E. J., Vermeersch, D. A., Nielsen, S. L., \& Smart, D. W. (2005). Is it time for clinicians to routinely track patient outcome? A 
meta-analysis. Clinical Psychology: Science and Practice, 10, 288-301. doi:10.1093/clipsy/bpg025

Lambert, M. J., Whipple, J. L., Smart, D. W., Vermeersch, D. A., Nielsen, S. L., \& Hawkins, E. J. (2001a). The effects of providing therapists with feedback on patient progress during psychotherapy: Are outcomes enhanced? Psychotherapy Research, 11, 49-68. doi:10.1080/713663852

Lambert, M. J., Whipple, J. L., Vermeersch, D. A., Smart, D. W., Hawkins, E. J., Nielson, S. L., \& Goates, M. (2002). Enhancing psychotherapy outcomes via providing feedback on client progress: A replication. Clinical Psychology and Psychotherapy, 9, 91-103. doi:10.1002/cpp.324

Lindgren, A., Barber, J. P., \& Sandahl, C. (2008). Alliance to the group-as-a-whole as a predictor of outcome in psychodynamic group therapy. International Journal of Group Psychotherapy, 58, 163-184. Retrieved from http://www.guilford.com/cgibin/cartscript.cgi?page=pr/jngr.htm\&dir=periodicals/ per_psych\&cart_id=460889.26343

Lipsey, M. W. (1990). Design sensitivity. Newbury Park, CA: Sage.

Locke, B. D., McAleavey, A.A., Zhao, Y., Lei, PW., Hayes, J.A., Castonguay, L.G., ... \& Lin, Y.C. (2012). Development and initial validation of the ounseling Center Assessment of Psychological Symptoms-34 (CCAPS-34). Measurement and Evaluation in Counseling and Development, 45, 151-169. doi: 10.1037/a0021282

Locke, B. D., Soet Buzolitz, J., Lei P. W., Boswell, J. F., McAleavey, A. A., Sevig, T. D., ... Hayes, J. A. (2011). Development of the Counseling Center Assessment of Psychological Symptoms-62 (CCAPS-62). Journal of Counseling Psychology, 58, 97-109. doi:10.1037/a0021282 
Locke, H. J., \& Wallace, K. M. (1959). Short marital adjustment and prediction tests: Their reliability and validity. Marriage and Family Living, 21, 251-255. doi: $10.2307 / 348022$

Luborsky, L. (1976). Helping alliances in psychotherapy. In J. L. Cleghorn (Ed.), Successful psychotherapy (pp. 92-116). New York, NY: Brunner.

Luborsky, L., Singer, B., \& Luborsky, L. (1975). Comparative studies of psychotherapies: Is it true that "Everyone has won and all must have prizes?" Archives of General Psychiatry, 32, 995-1008. Retrieved from http://archpsyc.ama-assn.org/

McCue, T., Carruthers, E., Dawe, J., Liu, S., Robar, A., \& Johnson, K. (2008). Evaluation of generalized linear model assumptions using randomization. Unpublished manuscript. Retrieved from http://www.mun.ca/biology/dschneider/b7932/B7932Final10Dec2008.pdf

MacKenzie, K. R. (1983). The clinical application of a group climate measure. In R. R. Dies \& K. R. MacKenzie (Eds.), Advances in group psychotherapy: Integrating research and practice (pp. 159-170). New York, NY: International Universities.

Martin, D. J., Garske, J. P., \& Davis, K. M. (2000). Relation of the therapeutic alliance with outcome variables: A meta-analytic review. Journal of Consulting and Clinical Psychology, 68, 438-450. doi:10.1037/0022-006X.68.3.438

Marziali, E., Munroe-Blum, H., \& McCleary, L. (1997). The contribution of group cohesion and group alliance to the outcome of group psychotherapy. International Journal of Group Psychotherapy, 47, 475-497. Retrieved from http://www.agpa.org/pubs/IJGPsubmit.html 
McKnight, P. E., McKnight, K. M., Sidani, S., \& Figueredo, A. J. (2007). Missing data: A gentle introduction. New York, NY: The Guilford.

McRoberts, C., Burlingame, G. M., \& Hoag, M. J. (1998). Comparative efficacy of individual and group psychotherapy: A meta-analytic perspective. Group Dynamics: Theory, Research, and Practice, 2, 101-117. doi:10.1037/10892699.2.2.101

Miller, S. D., \& Duncan, B. L. (2000). The outcome rating scale. Chicago, IL: Authors.

Miller, S. D., \& Duncan, B. L. (2004). The outcome and session rating scales: Administration and scoring manual. Ft. Lauderdale, FL: Authors.

Miller, S. D., Duncan, B. L., Brown, J., Sorrell, R., \& Chalk, B. (2006). Using formal client feedback to improve retention and outcome: Making ongoing, real-time assessment feasible. Journal of Brief Therapy, 5, 5-22. Retrieved from http://journalbrieftherapy.com/

Miller, S. D., Duncan, B. L., Brown, K., Sparks, J., \& Claud, D. (2003). The outcome rating scale: A preliminary study of the reliability, validity, and feasibility of a brief visual analog measure. Journal of Brief Therapy, 2, 91-100. Retrieved from http://journalbrieftherapy.com/

Miller, S. D., Duncan, B. L., \& Johnson, L. D. (2000). The session rating scale 3.0. Chicago, IL: Author.

Miller, S. D., Duncan, B. L., Sorrell, R., \& Brown, G. S. (2004). The partners for change outcome management system. Journal of Clinical Psychology, 61, 199-208. doi:10.1002/jclp.20111

Mintz, L. B., \& O’Halloran, M. S. (2000). The Eating Attitudes Test: Validation with DSM-IV eating disorder criteria. Journal of Personality 
Assessment, 74, 489-503. doi:10.1207/S15327752JPA7403_11

Myung, I. (2002). Tutorial on maximum likelihood estimation. Journal of Mathematical Psychology, 47, 90-100. doi: 10.1016/S0022-2496(02)00028-7

Newman, M. G., Kachin, K. E., Zuellig, A. R., Constantino, M. C., \& Cashman-McGrath, L. (2003). The Social Phobia Diagnostic Questionnaire: Preliminary validation of a new self-report diagnostic measure of social phobia. Psychological Medicine, 33, 623- 635. doi:10.1017/S0033291703007669

Norcross, J. C., \& Wampold, B. E. (2011). Evidenced-based therapy relationships: Research conclusions and clinical practices. Psychotherapy, 48, 98-102. doi:10.1037/a0022161

Ogrodniczuk, J. S., \& Piper, W. E. (2003). The effect of group climate on outcome in two forms of short-term group therapy. Group Dynamics: Theory, Research, and Practice, 7, 64-76. doi:10.1037/1089-2699.7.1.64

Piper, W. E., Ogrodniczuk, J. S., Lamarche, C., Hillscher, T., \& Joyce, A. S. (2005). Level of alliance, pattern of alliance, and outcome in short-term group therapy. International Journal of Group Therapy, 55, 527-550. Retrieved from http://www.guilford.com/cgibin/cartscript.cgi?page=pr/jngr.htm\&dir=periodicals/ per_psych\&cart_id=460889.26343

Raudenbush, S. W., \& Bryk, A. S. (2002). Hierarchical linear models: Applications and data analysis methods (6th ed.). Newbury Park, CA: Sage.

Raudenbush, S. W., Bryk, A. S, \& Congdon, R. (2004). HLM for windows (Version 6.0) [computer software]. Skokie, IL: Scientific Software International. 
Reese, R. J., Norsworthy, L. A., \& Rowlands, S. R. (2009). Does a continuous feedback model improve psychotherapy outcomes? Psychotherapy Theory, Research, Practice, Training, 46, 418-431. doi:10.1037/a0017901

Reese, R. J., Toland, M., Slone, N. C., \& Norsworthy, L. A. (2010). Effect of client feedback on couple psychotherapy outcome. Psychotherapy: Theory, Research, Practice, Training. 47, 616-630. doi:10.1037/a0021182

Ryum, T., Hagen, R., Nordahl, H. M., Vogel P. A., \& Stiles, T. C. (2009). Perceived group climate as a predictor of long-term outcome in a randomized controlled trial of cognitive behavioral group therapy for patients with comorbid psychiatric disorders. Behavioural and Cognitive Psychotherapy, 37, 497-510. doi:10.1017/S1352465809990208

Saunders, J. B., Aasland, O. G., Babor, T. F., de la Fuente, J. R., \& Grant, M. (1993). Development of the Alcohol Use Disorders Identification Test (AUDIT): WHO collaborative project on early detection of persons with harmful alcohol consumption-II. Addiction, 88, 791-804. doi:10.1111/j.1360-

0443.1993.tb02093.x

Seiss, M. (2010). Generalized linear models: 2010 LISA short course series [PowerPoint slides]. Retrieved from http://www.lisa.stat.vt.edu/sites/default/files/Generalized_Linear_Models_Present ation.pptx

Shimokawa, K., Lambert, M. J., \& Smart, D. W. (2010). Enhancing treatment outcome of patients at risk of treatment failure: Meta-analytic and mega-analytic review of psychotherapy quality assurance system. Journal of Consulting and Clinical Psychology, 78, 298-311. doi:10.1037/a0019247 
Slade, K., Lambert, M. J., Harmon, S. C., Smart, D. W., \& Bailey, R. (2008). Improving psychotherapy outcome: The use of immediate electronic feedback and revised clinical support tools. Clinical Psychology and Psychotherapy, 15, 287-203. doi:10.1002/cpp.594

Smith, M. L., Glass, G. V., \& Miller, T. (1980). The benefits of psychotherapy. Baltimore, MD: The Johns Hopkins University.

Snyder, D. K., Castellani, A. M., \& Whisman, M. A. (2006). Current status and future directions in couple therapy. Annual Review of Psychology, 57, 317-344. doi:10.1146/annurev.psych.56.091103.070154

Spielberger, C. D. (1999). STAXI-2: The State-Trait Anger Expression Inventory professional manual. Odessa, FL: Psychological Assessment Resources.

Strauss, B., Burlingame, G. M., \& Bormann, B. (2008). Using the CORE-R battery in group psychotherapy. Journal of Clinical Psychology, 64, 1225-1237. doi:10.1002/jclp.20535

Tasca, G. A., Illing, V., Joyce, A. S., \& Ogrodniczuk, J. S. (2009) Three-level multilevel growth models for nested change data: A guide for group treatment researchers, Psychotherapy Research, 19, 453-461. doi: 10.1080/10503300902933188

Taylor, N. T., Burlingame, G. M., Fuhriman, A., Johansen, J., \& Dahl, D. (2001). A survey of mental health care providers and managed care organization attitudes toward, familiarity with, and use of group interventions. International Journal of Group Therapy, 52, 243-263. doi:10.1521/ijgp.51.2.243.49848

Wampold, B. E. (2001). The great psychotherapy debate: Model, methods, and findings. Mahwah, NJ: Lawrence Erlbaum. 
Wampold, B. E., \& Brown, G. S. (2005). Estimating variability in outcomes attributable to therapists: A naturalistic study of outcomes in managed care. Journal of Consulting and Clinical Psychology, 73, 914-923. doi:10.1037/0022006X.73.5.914

Wampold, B. E., Mondin, G. W., Moody, M., Stich, F., Benson, K., \& Ahn, H. (1997). A meta-analysis of outcome studies comparing bona fide psychotherapies: Empirically, 'all must have prizes.' Psychological Bulletin, 122, 203-215. doi:10.1037/0033-2909.122.3.203

Whipple, J. L., Lambert, M. J., Vermeersch, D. A., Smart, D. W., Nielsen, S. L., \& Hawkins, E. J. (2003). Improving the effects of psychotherapy: The use of early identification of treatment failure and problem-solving strategies in routine practice. Journal of Counseling Psychology, 50, 59-68. doi:10.1037/00220167.50.1.59

Wierzbicki, M., \& Pekarik, G. (1993). A meta-analysis of psychotherapy drop out. Professional Psychology: Research and Practice, 24, 190-195. doi:10.1037/07357028.24.2.190

Xu, H. (2009). LOCF method and application in clinical data analysis. Proceedings published at National SAS Users Group Conference, Burlington VT. Retrieved from http://www.nesug.org/Proceedings/nesug09/po/po12.pdf

Yalom, I. D. (1995). The theory and practice of group psychotherapy $\left(4^{\text {th }}\right.$ ed.). New York, NY: Basic Books. 


\section{GENERAL INFORMATION}

Full Name: Norah Chapman Slone

\section{EDUCATION}

Doctor of Philosophy (Doctoral Candidate), Counseling Psychology

University of Kentucky, Lexington, KY

August 2008-Present

Expected date of graduation: August 2013

Specialties: Client feedback and Telepsychology

Dissertation Title: Evaluating the efficacy of client feedback in group psychotherapy

Dissertation Chair: Robert J. Reese, Ph.D.

Specialist in Education, Counseling Psychology

University of Kentucky, Lexington, KY

August 2008-May 2010

Master of Science, Counseling Psychology

Abilene Christian University, Abilene, TX

August 2006- May 2008

Bachelor of Science, Psychology and Bible

Kentucky Christian University

August 2002-May 2006

\section{PROFESSIONAL POSITIONS HELD}

\section{Clinical Experience}

Predoctoral Intern, University of North Carolina - Charlotte Counseling Center August 2012 - PRESENT, Charlotte, NC

Training Director: Theresa Rhodes, PhD

Practicum Student, Bureau of Prisons, Federal Medical Center-Women's Satellite Camp Fall 2011 - Spring 2012, Lexington, KY

Site Supervisor: Susan Masterson, $\mathrm{PhD}$ University Supervisor: Jeff Reese, $\mathrm{PhD}$

Intake Specialist and Parent Trainer, Telehealth Behavioral Family Intervention Project Summer 2009 - Spring 2012, Lexington, KY

Site Supervisor: Jeff Reese, PhD University Supervisor: Jeff Reese, PhD

Practicum Student Group Co-leader/Process Observer, University of Kentucky Counseling Center

Fall 2011- Spring 2012, Lexington, KY

Site Supervisor: Susan Mathews, PhD University Supervisor: Jeff Reese, PhD 
Supervisor of Master's Students, Clinical Supervision Course

February 2012 - April 2012, Lexington, KY

Site Supervisor: Jeff Reese, PhD University Supervisor: Jeff Reese, PhD

Supervisor of Master's Students, Clinical Supervision Course

February 2011 - April 2011, Lexington, KY

Site Supervisor: Sharon Rostosky, PhD University Supervisor: Keisha Love, PhD

Practicum Student-Psychology Affiliate, Eastern State Hospital (Inpatient Psychiatric Hospital),

Fall 2010 - July 2011, Lexington, KY

Site Supervisor: David Susman, PhD University Supervisors: Keisha Love, PhD/Jeff

Reese, $\mathrm{PhD}$

Practicum Student Counselor, University of Kentucky Counseling Center

Fall 2009 - Summer 2010, Lexington, KY

Site Supervisor: Di Sobel, PhD/Nathaniel Hopkins, PhD University Supervisor: Jeff

Reese, $\mathrm{PhD}$

Diversity Trainer, Co-leader, Gatton Group (University of Kentucky School of Business) Spring 2009, Lexington, KY

Site Supervisor: Pam Remer, $\mathrm{PhD} \quad$ University Supervisor: Jeff Reese, $\mathrm{PhD}$

Supervisor, Clinical Supervision Course

February 2009 - April 2009, Lexington, KY

Site Supervisor: Sharon Rostosky, PhD University Supervisor: Sharon Rostosky, PhD

Practicum Student, Chrysalis House (Residential Substance Abuse Treatment Facility)

Fall 2007, Lexington, KY

Site Supervisor: Carmella Yates, PhD University Supervisor: Larry Norsworthy, PhD

Practicum Student (Masters level), Shades of Hope (Residential Treatment Facility)

Summer 2007, Buffalo Gap, TX

Site Supervisor: Laurie Stevens-Beck, LMFT University Supervisor: Larry

Norsworthy, $\mathrm{PhD}$

Practicum Student (Masters level), Office of Career and Academic Development Spring 2007, Abilene, TX

Site Supervisor: Deon Botha, LMFT University Supervisor: Jeff Reese, PhD

Mental Health Associate, The Ridge Behavioral Health System (Inpatient Psychiatric

Hospital)

December 2005 - August 2006, Lexington, KY

Site Supervisor: Director of Nursing University Supervisor: N/A 


\section{Teaching Experience:}

Fall 2011: Guest Lecturer, University of Kentucky, Personality Assessment, Personality Assessment Inventory

Fall 2009-2011: Guest Lecturer, University of Kentucky, Theories of Counseling, Motivational Interviewing

Spring 2010: Co-Instructor, University of Kentucky, Principles of Psychological Assessment

Fall 2008: Lecturer and presenter, Bryan Station High School (Lexington, KY), Career Development

Summer 2007: Guest Lecturer, Abilene Christian University, Developmental Psychology, Moral Development

Spring 2007: Guest Lecturer, Abilene Christian University, Sports Psychology, Psychopathology

\section{Research Experience:}

Research Assistant, August 2010 - August 2012

Supervisor: Jeff Reese, $\mathrm{PhD}$

University of Kentucky, Department of Educational, School, and Counseling Psychology

Project: Using telehealth to reach underserved populations: A behavioral family intervention for parents of children with ADHD.

Research Assistant, August 2008 - August 2010

Supervisors: Dr. Ellen Hahn, DNS, Heather Robertson, MPH

University of Kentucky, College of Nursing

Project: Kentucky Center for Smoke Free Policy, Clean Indoor Air Partnership

Research Team Member, December 2009 - August 2010

Supervisor: Dr. Jeff Reese, $\mathrm{PhD}$

University of Kentucky, Department of Educational, School, and Counseling Psychology

Project: The reliability and convergent validity of the Targeted Individual Progress System (TIPS).

Research Team Member, January 2008 - December 2009

Supervisor: Dr. Jeff Reese, PhD

University of Kentucky, Department of Educational, School, and Counseling

Psychology

Project: Does using continuous feedback work for couples?

Research Team Member, August - December 2008

Supervisors: Keisha Love, $\mathrm{PhD}$ and Jeff Reese, $\mathrm{PhD}$

University of Kentucky, Department of Educational, School, and Counseling Psychology

Project: The effectiveness of career development interventions using the Kuder Career Assessment program. 
Graduate Assistant/Teaching Assistant, August 2006 - May 2007

Supervisor: Jeff Reese, $\mathrm{PhD}$

Abilene Christian University, Department of Psychology

Courses: Tests and Measurements, Sports Psychology

\section{PROFESSIONAL PUBLICATIONS AND PRESENTATIONS}

\section{Refereed Publications:}

Reese, R. J., Slone, N. C., Sprang, R., \& Soares, N. (2012). Telepsychology for Underserved Families: An Evidence-Based Parenting Program. Psychological Services, 9, 320-322. doi:10.1037/a0026193

Reese, R. J., Toland, M., Slone, N. C., \& Norsworthy, L. A. (2010). Effect of client feedback on couple psychotherapy outcomes. Psychotherapy: Theory, Research, Practice, Training, 47, 616-630. doi: 10.1037/a0021182

Reese, R. J., Slone, N. C., \& Miserocchi, K. (in press). Utilizing Client Feedback in Psychotherapy from an Interpersonal Process Perspective. Psychotherapy.

Slone, N. C., Reese, R. J., \& McClellan, M. J. (2012). Telepsychology Outcome Research with Children and Adolescents: A Review of the Literature. Psychological Services, 9, 272-292. doi:10.1037/a0027607

\section{Non-Refereed Publications}

Jones, L. K., Wagner, K., Darville, A., Slone, N., \& Hahn, E. J. (2009). Primary prevention of lung cancer in Appalachia through state and local policy change. Appalachian Community Cancer Network Policy Brief. Retrieved from http://www.mc.uky.edu/tobaccopolicy/ResearchProduct/policy.html

\section{Manuscripts in Progress}

Slone, N. C., Reese, R. J., Sprang, R., \& Soares, N. Using Telepsychology to Provide a Behavioral Family Intervention to Underserved Families: An Evaluation of Outcome and Feasibility. Will be submitted for review by February 1, 2013.

Slone, N. C., Reese, R. J., Prout, T. H., Grossl, A. B., Schultz, B., Newsome, B., Buschar, C., Perkins, A., Reliability and Validity of TIPS: A Brief Psychotherapy Outcome Measure. Will be submitted for review by February 1, 2013.

\section{Refereed Conference Presentations}

Slone, N. C., Grossl, A. B., Schultz, B., Newsome, B., Buschar, C., Perkins, A., Reese, R. J., \& Prout, T. H. (2012, August). Reliability and Validity of TIPS: A Brief Psychotherapy Outcome Measure. Poster presented at the Annual meeting of the American Psychological Association, Orlando, FL.

Slone, N. C., Reese, R. J., Mathews, S., Hopkins, N., \& Kodet, J. (2012, August). Using Client Feedback to Evaluate Group Psychotherapy Services: Pilot Findings. Poster presented at the Annual meeting of the American Psychological Association, Orlando, FL. 
Slone, N. C., Reese, R. J., Mathews, S., \& Hopkins, N. (2012, June). Evaluating the efficacy of client feedback in group psychotherapy: Preliminary findings. Accepted for poster presentation at the Annual International meeting of the Society for Psychotherapy Research, Virginia Beach, VA.

Slone, N. C., Reese, R. J., Sprang, R., \& Soares, N. (2011, September). Using Telehealth to Deliver Parent Training to Underserved Families with Children who have ADHD. Accepted for poster presentation at the Annual meeting of Society for Developmental \& Behavioral Pediatrics, San Antonio, TX.

Slone, N. C., Reese, R. J., Sprang, R., \& Soares, N. (2011, August). Using Telehealth to Reach Underserved Populations: A Behavioral Family Intervention for Parents of Children with Attention Deficit Hyperactivity Disorder (ADHD). Poster presented at the Annual meeting of the American Psychological Association, Washington, D.C.

Slone, N. C., Grossl, A. B., Schultz, B., \& Reese, R. J. (2010, October). Reliability and Validity of TIPS: A Brief Psychotherapy Outcome Measure. Paper presented at the Southeastern Regional Counseling Psychology Conference, Radford University.

Reese, R. J. \& Slone, N. C. (2009, August). Does continuous assessment improve psychotherapy outcome with couples? Poster presented at the Annual meeting of the American Psychological Association, Toronto, Ontario, Canada.

\section{Non-Refereed Presentations}

Panel representative ( 1 of 2 students) in the Counseling Psychology program. Advocating for education and opportunities to promote inclusion of students' diverse identities in the College Education. College of Education Faculty Meeting, Fall 2009. University of Kentucky.

\section{PROFESSIONAL AFFILIATIONS}

American Psychological Association Student Affiliate

Division 17 Student Affiliate

Division 35 Student Affiliate
Division 49 Student Affiliate

$$
\begin{aligned}
& 2008 \text { - PRESENT } \\
& 2008 \text { - PRESENT } \\
& 2010 \text { - PRESENT } \\
& 2012 \text { - PRESENT }
\end{aligned}
$$

Kentucky Psychological Association Student Affiliate 2008 - PRESENT

Society for Psychotherapy Research
2011 - PRESENT

\section{PROFESSIONAL SERVICE}

- Member at Large-Student Representative, APA Division 17 Section for the Promotion of Psychotherapy Science, October 2011- PRESENT

- American Psychological Association of Graduate Students (APAGS) Ambassador, American Psychological Association National Convention 2012 in Orlando FL, August 2012 
- American Psychological Association of Graduate Students (APAGS) Ambassador, American Psychological Association National Convention 2011 in Washington D.C., August 2011

- Committee Member, Tobacco Treatment and Addictions Committee, March 2009 November 2010

- Assistant Screener, Alcohol Screening Day Outreach; UK Counseling and Testing Center, April 2009

\section{AWARDS AND HONORS}

University of Kentucky, College of Education, Research Assistant, Fall 2010-2012 (\$12,000/year stipend)

University of Kentucky, Research Assistant, Fall 2008-Summer 2010 (\$14,000/year stipend)

Mental Health Association of Kentucky, Volunteer of the Year Award, Spring 2004 (\$1000 scholarship) 